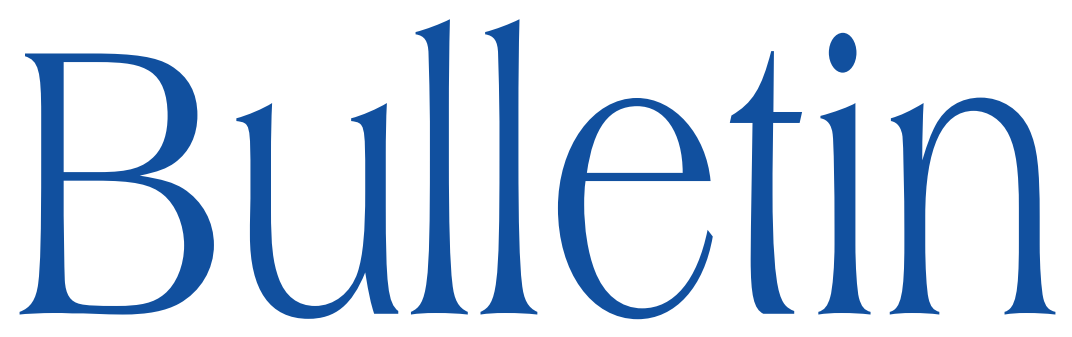

de la SOCIÉTÉ MATHÉMATIQUE DE FRANCE

\title{
ON THE STRUCTURE OF TRIANGULATED CATEGORIES
}

Claire Amiot

Tome 135

Fascicule 3

2007 


\title{
ON THE STRUCTURE OF TRIANGULATED CATEGORIES WITH FINITELY MANY INDECOMPOSABLES
}

\author{
By Claire Amiot
}

\begin{abstract}
We study the problem of classifying triangulated categories with finitedimensional morphism spaces and finitely many indecomposables over an algebraically closed field $k$. We obtain a new proof of the following result due to Xiao and Zhu: the Auslander-Reiten quiver of such a category $\mathcal{T}$ is of the form $\mathbb{Z} \Delta / G$ where $\Delta$ is a disjoint union of simply-laced Dynkin diagrams and $G$ a weakly admissible group of automorphisms of $\mathbb{Z} \Delta$. Then we prove that for 'most' groups $G$, the category $\mathcal{T}$ is standard, i.e. $k$-linearly equivalent to an orbit category $\mathcal{D}^{b}(\bmod k \Delta) / \Phi$. This happens in particular when $\mathcal{T}$ is maximal $d$-Calabi-Yau with $d \geq 2$. Moreover, if $\mathcal{T}$ is standard and algebraic, we can even construct a triangle equivalence between $\mathcal{T}$ and the corresponding orbit category. Finally we give a sufficient condition for the category of projectives of a Frobenius category to be triangulated. This allows us to construct non standard 1-Calabi-Yau categories using deformed preprojective algebras of generalized Dynkin type.
\end{abstract}

Texte reçu le 16 janvier 2007, accepté le $1^{\text {er }}$ juin 2007

Claire Amiot, Université Paris 7, Institut de Mathématiques de Jussieu, Théorie des groupes et des représentations, Case 7012, 2 Place Jussieu, 75251 Paris Cedex 05 (France) E-mail : amiot@math.jussieu.fr

2000 Mathematics Subject Classification. - 18E30, 16G70.

Key words and phrases. - Locally finite triangulated category, Calabi-Yau category, Dynkin diagram, Auslander-Reiten quiver, orbit category. 
RÉsumé (Sur la structure des catégories triangulées). - Cet article traite du problème de classification des catégories triangulées sur un corps algébriquement clos $k$ dont les espaces de morphismes sont de dimension finie et avec un nombre fini d'indécomposables. Nous obtenons une nouvelle preuve du résultat suivant dû à Xiao et Zhu : le carquois d'Auslander-Reiten d'une telle catégorie $\mathcal{T}$ est de la forme $\mathbb{Z} \Delta / G$ où $\Delta$ est une union disjointe de diagrammes de Dynkin simplement lacés et $G$ est un groupe d'automorphismes de $\mathbb{Z} \Delta$ faiblement admissible. Nous montrons ensuite que pour 'presque' tous groupes $G$, la catégorie $\mathcal{T}$ est standard, c'est-à-dire $k$-linéairement équivalente à une catégorie d'orbites $\mathcal{D}^{b}(\bmod k \Delta) / \Phi$. C'est en particulier le cas lorsque $\mathcal{T}$ est maximale $d$-Calabi-Yau avec $d \geq 2$. De plus, si $\mathcal{T}$ est standard et algébrique, nous pouvons même construire une équivalence triangulée entre $\mathcal{T}$ et la catégorie d'orbites correspondante. Nous donnons finalemant une condition suffisante pour que la catégorie de projectifs d'une catégorie de Frobenius soit triangulée. Cela nous permet de construire des catégories 1-Calabi-Yau non standard en utilisant les algèbres préprojectives déformées de type Dynkin généralisé.

\section{Introduction}

Let $k$ be an algebraically closed field and $\mathcal{T}$ a small Krull-Remak-Schmidt $k$-linear triangulated category (see [47]). We assume that

a) $\mathcal{T}$ is Hom-finite, i.e. the space $\operatorname{Hom}_{\mathcal{T}}(X, Y)$ is finite-dimensional for all objects $X, Y$ of $\mathcal{T}$.

It follows that indecomposable objects of $\mathcal{T}$ have local endomorphism rings and that each object of $\mathcal{T}$ decomposes into a finite direct sum of indecomposables [17, 3.3]. We assume moreover that

b) $\mathcal{T}$ is locally finite, i.e. for each indecomposable $X$ of $\mathcal{T}$, there are at most finitely many isoclasses of indecomposables $Y$ such that $\operatorname{Hom}_{\mathcal{T}}(X, Y) \neq 0$.

It was shown in [48] that condition $b$ ) implies its dual. Condition $b$ ) holds in particular if we have

$\left.\mathrm{b}^{\prime}\right) \mathcal{T}$ is additively finite, i.e. there are only finitely many isomorphism classes of indecomposables in $\mathcal{T}$.

The study of particular classes of such triangulated categories $\mathcal{T}$ has a long history. Let us briefly recall some of its highlights:

1) If $A$ is a representation-finite selfinjective algebra, then the stable category $\mathcal{T}$ of finite-dimensional (right) $A$-modules satisfies our assumptions and is additively finite. The structure of the underlying $k$-linear category of $\mathcal{T}$ was determined by C. Riedtmann in [39], [40], [41] and [42].

2) In [21], D. Happel showed that the bounded derived category of the category of finite-dimensional representations of a representation-finite quiver is locally finite and described its underlying $k$-linear category. 
3) The stable category $\underline{\mathrm{CM}}(R)$ of Cohen-Macaulay modules over a commutative complete local Gorenstein isolated singularity $R$ of dimension $d$ is a Hom-finite triangulated category which is $(d-1)$-Calabi-Yau (cf. for example [28] and [50]). In [4], M. Auslander and I. Reiten showed that if the dimension of $R$ is 1 , then the category $\underline{\operatorname{CM}}(R)$ is additively finite and computed the shape of the components of its Auslander-Reiten quiver.

4) The cluster category $\mathcal{C}_{Q}$ of a finite quiver $Q$ without oriented cycles was introduced in [12] if $Q$ is an orientation of a Dynkin diagram of type $\mathbb{A}$ and in [11] in the general case. The category $\mathcal{C}_{Q}$ is triangulated [30] and, if $Q$ is representation-finite, satisfies a) and $b^{\prime}$ ).

In a recent article [48], J. Xiao and B. Zhu determined the structure of the Auslander-Reiten quiver of a locally finite triangulated category. In this paper, we obtain the same result with a new proof in Section 4, namely that each connected component of the Auslander-Reiten quiver of the category $\mathcal{T}$ is of the form $\mathbb{Z} \Delta / G$, where $\Delta$ is a simply-laced Dynkin diagram and $G$ is trivial or a weakly admissible group of automorphisms. Contrary to J. Xiao and B. Zhu, we do not discuss separately the case where the Auslander-Reiten contains a loop.

We are interested in the $k$-linear structure of $\mathcal{T}$. If the Auslander-Reiten quiver of $\mathcal{T}$ is of the form $\mathbb{Z} \Delta$, we show that the category $\mathcal{T}$ is standard, i.e. it is equivalent to the mesh category $k(\mathbb{Z} \Delta)$. Then in Section 6 , we prove that $\mathcal{T}$ is standard if the number of vertices of $\Gamma=\mathbb{Z} \Delta / G$ is strictly greater than the number of isoclasses of indecomposables of $\bmod k \Delta$. In the last section, using [8] we construct examples of non standard triangulated categories such that $\Gamma=\mathbb{Z} \Delta / \tau$.

Finally, in the standard cases, we are interested in the triangulated structure of $\mathcal{T}$. For this, we need to make additional assumptions on $\mathcal{T}$. If the AuslanderReiten quiver is of the form $\mathbb{Z} \Delta$, and if $\mathcal{T}$ is the base of a tower of triangulated categories [29], we show that there is a triangle equivalence between $\mathcal{T}$ and the derived category $\mathcal{D}^{b}(\bmod k \Delta)$. For the additively finite cases, we have to assume that $\mathcal{T}$ is standard and algebraic in the sense of [31]. We then show that $\mathcal{T}$ is (algebraically) triangle equivalent to the orbit category of $\mathcal{D}^{b}(\bmod k \Delta)$ under the action of a weakly admissible group of automorphisms. In particular, for each $d \geq 2$, the algebraic triangulated categories with finitely many indecomposables which are maximal Calabi-Yau of CY-dimension $d$ are parametrized by the simply-laced Dynkin diagrams.

Our results apply in particular to many stable categories $\underline{\bmod } A$ of representation-finite selfinjective algebras $A$. These algebras were classified up to stable equivalence by C. Riedtmann [40], [42] and H. Asashiba [1]. In [9], J. Białkowski and A. Skowroński give a necessary and sufficient condition 
on these algebras so that their stable categories $\underline{\bmod } A$ are Calabi-Yau. In [26] and [27], T. Holm and P. Jørgensen prove that certain stable categories $\underline{\bmod } A$ are in fact $d$-cluster categories. These results can also be proved using our Corollary 7.3.

This paper is organized as follows: In Section 1, we prove that $\mathcal{T}$ has Auslander-Reiten triangles. Section 2 is dedicated to definitions about stable valued translation quivers and admissible automorphisms groups [23], [24], [14]. We show in Section 3 that the Auslander-Reiten quiver of $\mathcal{T}$ is a stable valued quiver and in Section 4, we reprove the result of J. Xiao and B. Zhu [48]: The Auslander-Reiten quiver is a disjoint union of quivers $\mathbb{Z} \Delta / G$, where $\Delta$ is a Dynkin quiver of type $\mathbb{A}, \mathbb{D}$ or $\mathbb{E}$, and $G$ a weakly admissible group of automorphisms. In Section 5, we construct a covering functor $\mathcal{D}^{b}(\bmod k \Delta) \rightarrow \mathcal{T}$ using Riedtmann's method [39]. Then, in Section 6, we exhibit some combinatorial cases in which $\mathcal{T}$ has to be standard, in particular when $\mathcal{T}$ is maximal $d$-Calabi-Yau with $d \geq 2$. Section 7 is dedicated to the algebraic case. If $\mathcal{T}$ is algebraic and standard, we can construct a triangle equivalence between $\mathcal{T}$ and an orbit category. If $\mathcal{P}$ is a $k$-category such that $\bmod \mathcal{P}$ is a Frobenius category satisfying certain conditions, we will prove in Section 8 that $\mathcal{P}$ has naturally a triangulated structure. This allows us to deduce in Section 9 that the category proj $P^{f}(\Delta)$ of the projective modules over a deformed preprojective algebra of generalized Dynkin type [8] is naturally triangulated and to reduce the classification of the additively finite triangulated categories which are 1-Calabi-Yau to that of the deformed preprojective algebras in the sense of [8]. In particular, thanks to [8], we obtain the existence of non standard 1Calabi-Yau categories in characteritic 2. Using our results and an extension of those of [8], Białkowski and Skowroński have recently proved [10] the existence of non standard 1-Calabi-Yau categories in characteristic 3. This is noteworthy since in characteristic different from 2, additively finite module categories are always standard [6].

Acknowledgments. - I would like to thank my supervisor B. Keller for his availability and many helpful discussions, and P. Jørgensen for his interest in this work and for suggesting some clarifications to me in the proof of Theorem 7.2.

\section{Notation and terminology}

We work over an algebraically closed field $k$. By a triangulated category, we mean a $k$-linear triangulated category $\mathcal{T}$. We write $S$ for the suspension functor of $\mathcal{T}$ and $U \stackrel{u}{\rightarrow} V \stackrel{v}{\rightarrow} W \stackrel{w}{\rightarrow} S U$ for a distinguished triangle. We say that $\mathcal{T}$ is Hom-finite if for each pair $X, Y$ of objects in $\mathcal{T}$, the space

TOME $135-2007-\mathrm{N}^{\mathrm{O}} 3$ 
$\operatorname{Hom}_{\mathcal{T}}(X, Y)$ is finite-dimensional over $k$. The category $\mathcal{T}$ will be called a KrullRemak-Schmidt category if each object is isomorphic to a finite direct sum of indecomposable objects with unicity (up to reordering) of this decomposition, and if the endomorphism ring of an indecomposable object is a local ring. This implies that idempotents of $\mathcal{T}$ split, i.e. if $e$ is an idempotent of $X$, then $e=\sigma \rho$ where $\sigma$ is a section and $\rho$ is a retraction [22, I, 3.2]. The category $\mathcal{T}$ will be called locally finite if for each indecomposable $X$ of $\mathcal{T}$, there are only finitely many isoclasses of indecomposables $Y$ such that $\operatorname{Hom}_{\mathcal{T}}(X, Y) \neq 0$. This property is selfdual by [48, prop. 1.1].

The Serre functor will be denoted by $\nu$ (see definition in Section 1). The Auslander-Reiten translation will always be denoted by $\tau$ (Section 1 ).

Let $\mathcal{T}$ and $\mathcal{T}^{\prime}$ be two triangulated categories. An $S$-functor $(F, \phi)$ is given by a $k$-linear functor $F: \mathcal{T} \rightarrow \mathcal{T}^{\prime}$ and a functor isomorphism $\phi$ between the functors $F \circ S$ and $S^{\prime} \circ F$, where $S$ is the suspension of $\mathcal{T}$ and $S^{\prime}$ the suspension of $\mathcal{T}^{\prime}$. The notion of $\nu$-functor, or $\tau$-functor is then clear. A triangle functor is an $S$-functor $(F, \phi)$ such that for each triangle $U \stackrel{u}{\rightarrow} V \stackrel{v}{\rightarrow} W \stackrel{w}{\rightarrow} S U$ of $\mathcal{T}$, the sequence $F U \stackrel{F u}{\longrightarrow} F V \stackrel{F v}{\longrightarrow} F W \stackrel{\phi_{U} \circ F w}{\longrightarrow} S^{\prime} F U$ is a triangle of $\mathcal{T}^{\prime}$.

The category $\mathcal{T}$ is Calabi-Yau if there exists an integer $d>0$ such that we have a triangle functor isomorphism between $S^{d}$ and $\nu$. We say that $\mathcal{T}$ is maximal $d$-Calabi-Yau if $\mathcal{T}$ is $d$-Calabi-Yau and if for each covering functor $\mathcal{T}^{\prime} \rightarrow \mathcal{T}$ with $\mathcal{T}^{\prime} d$-Calabi-Yau, we have a $k$-linear equivalence between $\mathcal{T}$ and $\mathcal{T}^{\prime}$.

For an additive $k$-category $\mathcal{E}$, we write $\bmod \mathcal{E}$ for the category of contravariant finitely presented functors from $\mathcal{E}$ to $\bmod k$ (Section 8 ), and if the projectives of $\bmod \mathcal{E}$ coincide with the injectives, $\underline{\bmod \mathcal{E}}$ will be the stable category.

\section{Serre duality and Auslander-Reiten triangles}

1.1. Serre duality. - Recall from [38] that a Serre functor for $\mathcal{T}$ is an autoequivalence $\nu: \mathcal{T} \rightarrow \mathcal{T}$ together with an isomorphism $D \operatorname{Hom}_{\mathcal{T}}(X, ?) \simeq$ $\operatorname{Hom}_{\mathcal{T}}(?, \nu X)$ for each $X \in \mathcal{T}$, where $D$ is the duality $\operatorname{Hom}_{k}(?, k)$.

TheOREM 1.1. - Let $\mathcal{T}$ be a Krull-Remak-Schmidt, locally finite triangulated category. Then $\mathcal{T}$ has a Serre functor $\nu$.

Proof. - Let $X$ be an object of $\mathcal{T}$. We write $X^{\wedge}$ for the functor $\operatorname{Hom}_{\mathcal{T}}($ ?, $X)$ and $F$ for the functor $D \operatorname{Hom}_{\mathcal{T}}(X, ?)$. Using the lemma [38, I.1.6] we just have to show that $F$ is representable. Indeed, the category $\mathcal{T}^{\text {op }}$ is locally finite as well. The proof is in two steps.

Step 1: The functor $F$ is finitely presented. - Let $Y_{1}, \ldots, Y_{r}$ be representatives of the isoclasses of indecomposable objects of $\mathcal{T}$ such that $F Y_{i}$ is not zero. The space $\operatorname{Hom}\left(Y_{i}^{\wedge}, F\right)$ is finite-dimensional over $k$. Indeed it is isomorphic 
to $F Y_{i}$ by the Yoneda lemma. Therefore, the functor $\operatorname{Hom}\left(Y_{i}^{\wedge}, F\right) \otimes_{k} Y_{i}^{\wedge}$ is representable. We get an epimorphism from a representable functor to $F$ :

$$
\bigoplus_{i=1}^{r} \operatorname{Hom}\left(Y_{i}^{\wedge}, F\right) \otimes_{k} Y_{i}^{\wedge} \longrightarrow F
$$

By applying the same argument to its kernel we get a projective presentation of $F$ of the form $U^{\wedge} \rightarrow V^{\wedge} \rightarrow F \rightarrow 0$, with $U$ and $V$ in $\mathcal{T}$.

Step 2: A cohomological functor $H: \mathcal{T}^{\mathrm{op}} \rightarrow \bmod k$ is representable if and only if it is finitely presented. - Let

$$
U^{\wedge} \stackrel{u^{\wedge}}{\longrightarrow} V^{\wedge} \stackrel{\phi}{\rightarrow} H \rightarrow 0
$$

be a presentation of $H$. We form a triangle $U \stackrel{u}{\rightarrow} V \stackrel{v}{\rightarrow} W \stackrel{w}{\rightarrow} S U$. We get an exact sequence

$$
U^{\wedge} \stackrel{u^{\wedge}}{\longrightarrow} V^{\wedge} \stackrel{v^{\wedge}}{\longrightarrow} W^{\wedge} \stackrel{w^{\wedge}}{\longrightarrow}(S U)^{\wedge}
$$

Since the composition of $\phi$ with $u^{\wedge}$ is zero and $H$ is cohomological, the morphism $\phi$ factors through $v^{\wedge}$. But $H$ is the cokernel of $u^{\wedge}$, so $v^{\wedge}$ factors through $\phi$. We obtain a commutative diagram

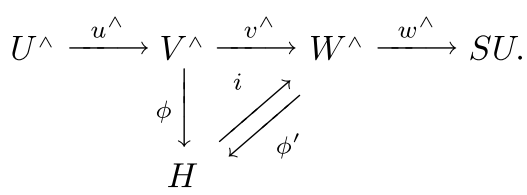

The equality $\phi^{\prime} \circ i \circ \phi=\phi^{\prime} \circ v^{\wedge}=\phi$ implies that $\phi^{\prime} \circ i$ is the identity of $H$ because $\phi$ is an epimorphism. We deduce that $H$ is a direct factor of $W^{\wedge}$. The composition $i \circ \phi^{\prime}=e^{\wedge}$ is an idempotent. Then $e \in \operatorname{End}(W) \operatorname{splits}$ and we get $H=W^{\prime \wedge}$ for a direct factor $W^{\prime}$ of $W$.

\subsection{Auslander-Reiten triangles}

Definition 1.2 .1 (see [21]). - A triangle $X \stackrel{u}{\rightarrow} Y \stackrel{v}{\rightarrow} Z \stackrel{w}{\rightarrow} S X$ of $\mathcal{T}$ is called an Auslander-Reiten triangle or AR-triangle if the following conditions are satisfied:

(AR1) $X$ and $Z$ are indecomposable objects;

(AR2) $w \neq 0$

(AR3) if $f: W \rightarrow Z$ is not a retraction, there exists $f^{\prime}: W \rightarrow Y$ such that $v f^{\prime}=f$

$\left(\mathrm{AR}^{\prime}\right)$ if $g: X \rightarrow V$ is not a section, there exists $g^{\prime}: Y \rightarrow V$ such that $g^{\prime} u=g$. 
Let us recall that, if (AR1) and (AR2) hold, the conditions (AR3) and (AR3') are equivalent. We say that a triangulated category $\mathcal{T}$ has Auslander-Reiten triangles if, for any indecomposable object $Z$ of $\mathcal{T}$, there exists an AR-triangle ending at $Z: X \stackrel{u}{\rightarrow} Y \stackrel{v}{\rightarrow} Z \stackrel{w}{\rightarrow} S X$. In this case, the AR-triangle is unique up to triangle isomorphism inducing the identity of $Z$.

The following proposition is proved in [38, Prop. I.2.3].

Proposition 1.2. - Let $\mathcal{T}$ be a Krull-Remak-Schmidt, locally finite triangulated category. Then the category $\mathcal{T}$ has Auslander-Reiten triangles.

The composition $\tau=S^{-1} \nu$ is called the Auslander-Reiten translation. An AR-triangle of $\mathcal{T}$ ending at $Z$ has the form

$$
\tau Z \stackrel{u}{\rightarrow} Y \stackrel{v}{\rightarrow} Z \stackrel{w}{\rightarrow} \nu Z .
$$

\section{Valued translation quivers and automorphism groups}

2.1. Translation quivers. - We recall some definitions and notations concerning quivers [14]. A quiver $Q=\left(Q_{0}, Q_{1}, s, t\right)$ is given by the set $Q_{0}$ of its vertices, the set $Q_{1}$ of its arrows, a source map $s$ and a tail map $t$. If $x \in Q_{0}$ is a vertex, we denote by $x^{+}$the set of direct successors of $x$, and by $x^{-}$the set of its direct predecessors. We say that $Q$ is locally finite if for each vertex $x \in Q_{0}$, there are finitely many arrows ending at $x$ and starting at $x$ (in this case, $x^{+}$and $x^{-}$are finite sets). The quiver $Q$ is said to be without double arrows, if two different arrows cannot have the same tail and source.

Definition 2.1.1. - A stable translation quiver $(Q, \tau)$ is a locally finite quiver without double arrows with a bijection $\tau: Q_{0} \rightarrow Q_{0}$ such that

$$
(\tau x)^{+}=x^{-} \text {for each vertex } x \text {. }
$$

For each arrow $\alpha: x \rightarrow y$, let $\sigma \alpha$ be the unique arrow $\tau y \rightarrow x$.

Note that a stable translation quiver can have loops.

DeFinition 2.1.2. - A valued translation quiver $(Q, \tau, a)$ is a stable translation quiver $(Q, \tau)$ with a map $a: Q_{1} \rightarrow \mathbb{N}$ such that

$$
a(\alpha)=a(\sigma \alpha) \text { for each arrow } \alpha .
$$

If $\alpha$ is an arrow from $x$ to $y$, we write $a_{x y}$ instead of $a(\alpha)$.

Definition 2.1.3. - Let $\Delta$ be an oriented tree. The repetition of $\Delta$ is the quiver $\mathbb{Z} \Delta$ defined as follows:

- $(\mathbb{Z} \Delta)_{0}=\mathbb{Z} \times \Delta_{0}$, 
- $(\mathbb{Z} \Delta)_{1}=\mathbb{Z} \times \Delta_{1} \cup \sigma\left(\mathbb{Z} \times \Delta_{1}\right)$ with arrows

$$
(n, \alpha):(n, x) \longrightarrow(n, y) \quad \text { and } \quad \sigma(n, \alpha):(n-1, y) \rightarrow(n, x)
$$

for each arrow $\alpha: x \rightarrow y$ of $\Delta$.

The quiver $\mathbb{Z} \Delta$ with the translation $\tau(n, x)=(n-1, x)$ is clearly a stable translation quiver which does not depend (up to isomorphism) on the orientation of $\Delta$ (see [39]).

\subsection{Groups of weakly admissible automorphisms}

Definition 2.2.1. - An automorphism group $G$ of a quiver is said to be admissible [39] if no orbit of $G$ intersects a set of the form $\{x\} \cup x^{+}$or $\{x\} \cup x^{-}$in more than one point. It said to be weakly admissible [14] if, for each $g \in G-\{1\}$ and for each $x \in Q_{0}$, we have $x^{+} \cap(g x)^{+}=\varnothing$.

Note that an admissible automorphism group is a weakly admissible automorphism group. Let us fix a numbering and an orientation of the simply-laced Dynkin trees.

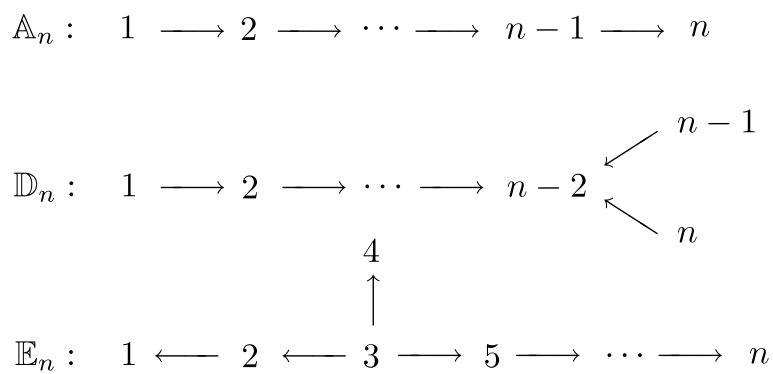

Let $\Delta$ be a Dynkin tree. We define an automorphism $S$ of $\mathbb{Z} \Delta$ as follows:

— if $\Delta=\mathbb{A}_{n}$, then $S(p, q)=(p+q, n+1-q)$;

— if $\Delta=\mathbb{D}_{n}$ with $n$ even, then $S=\tau^{-n+1}$;

— if $\Delta=\mathbb{D}_{n}$ with $n$ odd, then $S=\tau^{-n+1} \phi$ where $\phi$ is the automorphism of $\mathbb{D}_{n}$ which exchanges $n$ and $n-1$;

- if $\Delta=\mathbb{E}_{6}$, then $S=\phi \tau^{-6}$ where $\phi$ is the automorphism of $\mathbb{E}_{6}$ which exchanges 2 and 5 , and 1 and 6 ;

— if $\Delta=\mathbb{E}_{7}$, then $S=\tau^{-9}$;

— and if $\Delta=\mathbb{E}_{8}$, then $S=\tau^{-15}$.

In [39, Anhang 2], Riedtmann describes all admissible automorphism groups of Dynkin diagrams. Here is a more precise result in which we describe all weakly admissible automorphism groups of Dynkin diagrams. 
Theorem 2.1. - Let $\Delta$ be a Dynkin tree and $G$ a non trivial group of weakly admissible automorphisms of $\mathbb{Z} \Delta$. Then $G$ is isomorphic to $\mathbb{Z}$, and here is a list of its possible generators:

- if $\Delta=\mathbb{A}_{n}$ with $n$ odd, possible generators are $\tau^{r}$ and $\phi \tau^{r}$ with $r \geq 1$, where $\phi=\tau^{\frac{1}{2}(n+1)} S$ is an automorphism of $\mathbb{Z} \Delta$ of order 2 ;

- if $\Delta=\mathbb{A}_{n}$ with $n$ even, then possible generators are $\rho^{r}$, where $r \geq 1$ and where $\rho=\tau^{\frac{1}{2} n} S$ ( since $\rho^{2}=\tau^{-1}, \tau^{r}$ is a possible generator $)$;

- if $\Delta=\mathbb{D}_{n}$ with $n \geq 5$, then possible generators are $\tau^{r}$ and $\tau^{r} \phi$, where $r \geq 1$ and where $\phi=(n-1, n)$ is the automorphism of $\mathbb{D}_{n}$ exchanging $n$ and $n-1$;

- if $\Delta=\mathbb{D}_{4}$, then possible generators are $\phi \tau^{r}$, where $r \geq 1$ and where $\phi$ belongs to $\mathfrak{S}_{3}$ the permutation group on three elements seen as subgroup of automorphisms of $\mathbb{D}_{4}$;

- if $\Delta=\mathbb{E}_{6}$, then possible generators are $\tau^{r}$ and $\phi \tau^{r}$, where $r \geq 1$ and where $\phi$ is the automorphism of $\mathbb{E}_{6}$ exchanging 2 and 5 , and 1 and 6 ;

- if $\Delta=\mathbb{E}_{n}$ with $n=7,8$, possible generators are $\tau^{r}$, where $r \geq 1$.

The unique weakly admissible automorphism group which is not admissible exists for $\mathbb{A}_{n}, n$ even, and is generated by $\rho$.

\section{Property of the Auslander-Reiten translation}

We define the Auslander-Reiten quiver $\Gamma_{\mathcal{T}}$ of the category $\mathcal{T}$ as a valued quiver $(\Gamma, a)$. The vertices are the isoclasses of indecomposable objects. Given two indecomposable objects $X$ and $Y$ of $\mathcal{T}$, we draw one arrow from $x=[X]$ to $y=[Y]$ if the vector space $\mathcal{R}(X, Y) / \mathcal{R}^{2}(X, Y)$ is not zero, where $\mathcal{R}(?, ?)$ is the radical of the bifunctor $\operatorname{Hom}_{\mathcal{T}}(?, ?)$. A morphism of $\mathcal{R}(X, Y)$ which does not vanish in the quotient $\mathcal{R}(X, Y) / \mathcal{R}^{2}(X, Y)$ will be called irreducible. Then we put

$$
a_{x y}=\operatorname{dim}_{k} \mathcal{R}(X, Y) / \mathcal{R}^{2}(X, Y) .
$$

Remark that the fact that $\mathcal{T}$ is locally finite implies that its AR-quiver is locally finite. The aim of this section is to show that $\Gamma_{\mathcal{T}}$ with the translation $\tau$ defined in the first part is a valued translation quiver. In other words, we want to show the proposition:

Proposition 3.1. - If $X$ and $Y$ are indecomposable objects of $\mathcal{T}$, we have

$$
\operatorname{dim}_{k} \mathcal{R}(X, Y) / \mathcal{R}^{2}(X, Y)=\operatorname{dim}_{k} \mathcal{R}(\tau Y, X) / \mathcal{R}^{2}(\tau Y, X) .
$$

Let us recall some definitions [22].

Definition 3.0.2. - A morphism $g: Y \rightarrow Z$ is called sink morphism if the following hold 
1) $g$ is not a retraction;

2) if $h: M \rightarrow Z$ is not a retraction, then $h$ factors through $g$;

3) if $u$ is an endomorphism of $Y$ which satisfies $g u=g$, then $u$ is an automorphism.

Dually, a morphism $f: X \rightarrow Y$ is called source morphism if the following hold:

1) $f$ is not a section;

2) if $h: X \rightarrow M$ is not a section, then $h$ factors through $f$;

3 ) if $u$ is an endomorphism of $Y$ which satisfies $u f=f$, then $u$ is an automorphism.

These conditions imply that $X$ and $Z$ are indecomposable. Obviously, if $X \stackrel{u}{\rightarrow} Y \stackrel{v}{\rightarrow} Z \stackrel{w}{\rightarrow} S X$ is an AR-triangle, then $u$ is a source morphism and $v$ is a sink morphism. Conversely, if $v \in \operatorname{Hom}_{\mathcal{T}}(Y, Z)$ is a sink morphism (or if $u \in \operatorname{Hom}_{\mathcal{T}}(X, Y)$ is a source morphism), then there exists an AR-triangle $X \stackrel{u}{\rightarrow} Y \stackrel{v}{\rightarrow} Z \stackrel{w}{\longrightarrow} S X$ (see [22, I, 4.5]).

The following lemma (and the dual statement) is proved in [43, 2.2.5].

LEMMA 3.2. - Let $g$ be a morphism from $Y$ to $Z$, where $Z$ is indecomposable and $Y=\bigoplus_{i=1}^{r} Y_{i}^{n_{i}}$ is the decomposition of $Y$ into indecomposables. Then the morphism $g$ is a sink morphism if and only if the following hold:

1) For each $i=1, \ldots, r$ and $j=1, \ldots, n_{i}$, the restriction $g_{i, j}$ of $g$ to the $j$-th component of the $i$-th isotopic part of $Y$ belongs to the radical $\mathcal{R}\left(Y_{i}, Z\right)$.

2) For each $i=1, \ldots, r$, the family $\left(\bar{g}_{i, j}\right)_{j=1, \ldots, n_{i}}$ forms a $k$-basis of the space $\mathcal{R}\left(Y_{i}, Z\right) / \mathcal{R}^{2}\left(Y_{i}, Z\right)$.

3) If $h \in \operatorname{Hom}_{\mathcal{T}}\left(Y^{\prime}, Z\right)$ is irreducible and $Y^{\prime}$ indecomposable, then $h$ factors through $g$ and $Y^{\prime}$ is isomorphic to $Y_{i}$ for some $i$.

Using this lemma, it is easy to see that Proposition 3.1 holds. Thus, the Auslander-Reiten quiver $\Gamma_{\mathcal{T}}=(\Gamma, \tau, a)$ of the category $\mathcal{T}$ is a valued translation quiver.

\section{Structure of the Auslander-Reiten quiver}

This section is dedicated to another proof of a theorem due to J. Xiao and B. Zhu:

Theorem 4.1 (see [49]). - Let $\mathcal{T}$ be a Krull-Remak-Schmidt, locally finite triangulated category. Let $\Gamma$ be a connected component of the AR-quiver of $\mathcal{T}$. Then there exists a Dynkin tree $\Delta$ of type $\mathbb{A}, \mathbb{D}$ or $\mathbb{E}$, a weakly admissible automorphism group $G$ of $\mathbb{Z} \Delta$ and an isomorphism of valued translation quivers

$$
\theta: \Gamma \stackrel{\sim}{\longrightarrow} \mathbb{Z} \Delta / G
$$

TOME $135-2007-\mathrm{N}^{\mathrm{O}} 3$ 
The underlying graph of the tree $\Delta$ is unique up to isomorphism (it is called the type of $\Gamma$ ), and the group $G$ is unique up to conjugacy in $\operatorname{Aut}(\mathbb{Z} \Delta)$. In particular, if $\mathcal{T}$ has an infinite number of isoclasses of indecomposable objects, then $G$ is trivial, and $\Gamma$ is the repetition quiver $\mathbb{Z} \Delta$.

4.1. Auslander-Reiten quivers with a loop. - In this section, we suppose that the Auslander-Reiten quiver of $\mathcal{T}$ contains a loop, i.e. there exists an arrow with same tail and source. Thus, we suppose that there exists an indecomposable $X$ of $\mathcal{T}$ such that

$$
\operatorname{dim}_{k} \mathcal{R}(X, X) / \mathcal{R}^{2}(X, X) \geq 1
$$

Proposition 4.2. - Let $X$ be an indecomposable object of $\mathcal{T}$. Suppose that we have $\operatorname{dim}_{k} \mathcal{R}(X, X) / \mathcal{R}^{2}(X, X) \geq 1$. Then $\tau X$ is isomorphic to $X$.

To prove this, we need a lemma.

LEMmA 4.3. - Let

$$
X_{1} \stackrel{f_{1}}{\longrightarrow} X_{2} \stackrel{f_{2}}{\longrightarrow} \cdots \stackrel{f_{n}}{\longrightarrow} X_{n+1}
$$

be a sequence of irreducible morphisms between indecomposable objects with $n \geq 2$. If the composition $f_{n} \circ f_{n-1} \circ \cdots \circ f_{1}$ is zero, then there exists an $i$ such that $\tau^{-1} X_{i}$ is isomorphic to $X_{i+2}$.

Proof. - The proof proceeds by induction on $n$. Let us show the assertion for $n=2$. Suppose $X_{1} \stackrel{f_{1}}{\longrightarrow} X_{2} \stackrel{f_{2}}{\longrightarrow} X_{3}$ is a sequence such that $f_{2} \circ f_{1}=0$. We can then construct an AR-triangle:

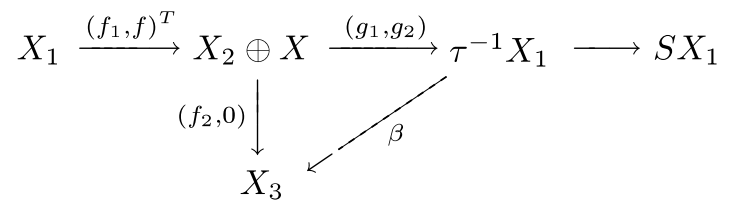

The composition $f_{2} \circ f_{1}$ is zero, thus the morphism $f_{2}$ factors through $g_{1}$. As the morphisms $g_{1}$ and $f_{2}$ are irreducible, we conclude that $\beta$ is a retraction, and $X_{3}$ a direct summand of $\tau^{-1} X_{1}$. But $X_{1}$ is indecomposable, so $\beta$ is an isomorphism between $X_{3}$ and $\tau^{-1} X_{1}$.

Now suppose that the property holds for an integer $n-1$ and that we have $f_{n} \circ f_{n-1} \circ \cdots \circ f_{1}=0$. If the composition $f_{n-1} \circ \cdots \circ f_{1}$ is zero, the proposition holds by induction. So we can suppose that for $i \leq n-2$, the objects $\tau^{-1} X_{i}$ and $X_{i+2}$ are not isomorphic. We show now by induction on $i$ that for each $i \leq n-1$, there exists a map $\beta_{i}: \tau^{-1} X_{i} \rightarrow X_{n+1}$ such 
that $f_{n} \circ \cdots \circ f_{i+1}=\beta_{i} g_{i}$ where $g_{i}: X_{i+1} \rightarrow \tau^{-1} X_{i}$ is an irreducible morphism. For $i=1$, we construct an AR-triangle:

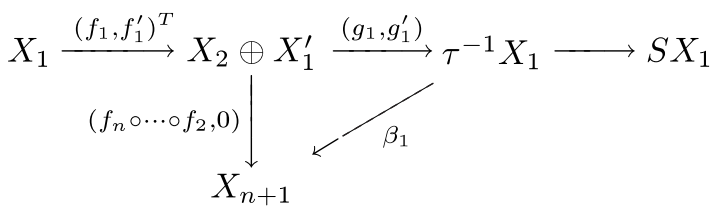

As the composition $f_{n} \circ \cdots \circ f_{1}$ is zero, we have the factorization $f_{n} \circ \cdots \circ f_{2}=$ $\beta_{1} g_{1}$.

Now for $i$, as $\tau^{-1} X_{i-1}$ is not isomorphic to $X_{i+1}$, there exists an AR-triangle of the form

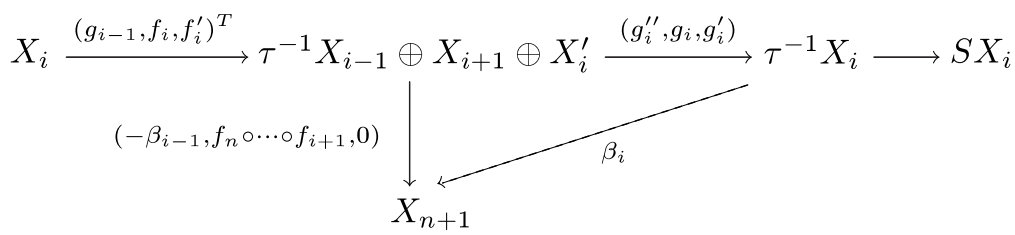

By induction, $-\beta_{i-1} g_{i-1}+f_{n} \circ \cdots \circ f_{i+1} f_{i}$ is zero, thus $f_{n} \circ \cdots \circ f_{i+1}$ factors through $g_{i}$. This property is true for $i=n-1$, so we have a map $\beta_{n-1}$ : $\tau^{-1} X_{n-1} \rightarrow X_{n+1}$ such that $\beta_{n-1} g_{n-1}=f_{n}$. As $g_{n-1}$ and $f_{n}$ are irreducible, we conclude that $\beta_{n-1}$ is an isomorphism between $X_{n+1}$ and $\tau^{-1} X_{n-1}$.

Now we are able to prove Proposition 4.2. There exists an irreducible map $f: X \rightarrow X$. Suppose that $X$ and $\tau X$ are not isomorphic. Then from the previous lemma, the endomorphism $f^{n}$ is non zero for each $n$. But since $\mathcal{T}$ is a Krull-Remak-Schmidt, locally finite category, a power of the radical $\mathcal{R}(X, X)$ vanishes. This is a contradiction.

4.2. Proof of Theorem 4.1. - Let $\widetilde{\Gamma}=\left(\widetilde{\Gamma}_{0}, \widetilde{\Gamma}_{1}, \tilde{a}\right)$ be the valued quiver obtained from $\Gamma$ by removing the loops, i.e. we have

$$
\widetilde{\Gamma}_{0}=\Gamma_{0}, \quad \widetilde{\Gamma}_{1}=\left\{\alpha \in \Gamma_{1} \text { such that } s(\alpha) \neq t(\alpha)\right\}, \quad \text { and } \quad \tilde{a}=a_{\mid \tilde{\Gamma}_{1}} .
$$

Lemma 4.4. - The quiver $\widetilde{\Gamma}=\left(\widetilde{\Gamma}_{0}, \widetilde{\Gamma}_{1}, \tilde{a}\right)$ with the translation $\tau$ is a valued translation quiver without loop.

Proof. - We have to check that the map $\sigma$ is well-defined. But from Proposition 4.2 , if $\alpha$ is a loop on a vertex $x, \sigma(\alpha)$ is the unique arrow from $\tau x=x$ to $x$, i.e. $\sigma(\alpha)=\alpha$. Thus $\widetilde{\Gamma}$ is obtained from $\Gamma$ by removing some $\sigma$-orbits and it keeps the structure of stable valued translation quiver.

TOME $135-2007-\mathrm{N}^{\mathrm{O}} 3$ 
Now, we can apply Riedtmann's Struktursatz [39] and the result of HappelPreiser-Ringel [24]. There exist a tree $\Delta$ and an admissible automorphism group $G$ (which may be trivial) of $\mathbb{Z} \Delta$ such that $\widetilde{\Gamma}$ is isomorphic to $\mathbb{Z} \Delta / G$ as a valued translation quiver. The underlying graph of the tree $\Delta$ is then unique up to isomorphism and the group $G$ is unique up to conjugacy in $\operatorname{Aut}(\mathbb{Z} \Delta)$. Let $x$ be a vertex of $\Delta$. We write $\bar{x}$ for the image of $x$ by the map:

$$
\Delta \rightarrow \mathbb{Z} \Delta \stackrel{\pi}{\rightarrow} \mathbb{Z} \Delta / G \simeq \widetilde{\Gamma} \longrightarrow \Gamma .
$$

Let $C: \Delta_{0} \times \Delta_{0} \rightarrow \mathbb{Z}$ be the matrix defined as follows:

- $C(x, y)=-a_{\bar{x} \bar{y}}$ (resp. $-a_{\bar{y}} \bar{x}$ ) if there exists an arrow from $x$ to $y$ (resp. from $y$ to $x)$ in $\Delta$,

- $C(x, x)=2-a_{\bar{x}} \bar{x}$

- $C(x, y)=0$ otherwise.

The matrix $C$ is symmetric; it is a 'generalized Cartan matrix' in the sense of [23]. If we remove the loops from the 'underlying graph of $C$ ' (in the sense of [23]), we get the underlying graph of $\Delta$.

In order to apply the result of Happel-Preiser-Ringel [23, Section 2], we have to show:

LEMMA 4.5. - The set $\Delta_{0}$ of vertices of $\Delta$ is finite.

Proof. - Riedtmann's construction of $\Delta$ is the following. We fix a vertex $x_{0}$ in $\widetilde{\Gamma}_{0}$. Then the vertices of $\Delta$ are the paths of $\widetilde{\Gamma}$ beginning on $x_{0}$ and which do not contain subpaths of the form $\alpha \sigma(\alpha)$, where $\alpha$ is in $\widetilde{\Gamma}_{1}$. Now suppose that $\Delta_{0}$ is an infinite set. Then for each $n$, there exists a sequence

$$
x_{0} \stackrel{\alpha_{1}}{\longrightarrow} x_{1} \stackrel{\alpha_{2}}{\longrightarrow} \cdots \stackrel{\alpha_{n-1}}{\longrightarrow} x_{n-1} \stackrel{\alpha_{n}}{\longrightarrow} x_{n}
$$

such that $\tau x_{i+2} \neq x_{i}$. Then there exist some indecomposables $X_{0}, \ldots, X_{n}$ such that the vector space $\mathcal{R}\left(X_{i-1}, X_{i}\right) / \mathcal{R}^{2}\left(X_{i-1}, X_{i}\right)$ is not zero. Thus from Lemma 4.3 , there exists irreducible morphisms $f_{i}: X_{i-1} \rightarrow X_{i}$ such that the composition $f_{n} \circ f_{n-1} \circ \cdots \circ f_{1}$ does not vanish. But the functor $\operatorname{Hom}_{\mathcal{T}}\left(X_{0}, ?\right)$ has finite support. Thus there is an indecomposable $Y$ which appears an infinite number of times in the sequence $\left(X_{i}\right)_{i}$. But since $\mathcal{R}^{N}(Y, Y)$ vanishes for an $N$, we have a contradiction.

Let $\mathcal{S}$ a system of representatives of isoclasses of indecomposables of $\mathcal{T}$. For an indecomposable $Y$ of $\mathcal{T}$, we put

$$
\ell(Y)=\sum_{M \in \mathcal{S}} \operatorname{dim}_{k} \operatorname{Hom}_{\mathcal{T}}(M, Y) .
$$

This sum is finite since $\mathcal{T}$ is locally finite. 
Lemma 4.6. - For $x$ in $\Delta_{0}$, we write $d_{x}=\ell(\bar{x})$. Then for each $x \in \Delta_{0}$, we have

$$
\sum_{y \in \Delta_{0}} d_{y} C_{x y}=2
$$

Proof. — Let $X$ and $U$ be indecomposables of $\mathcal{T}$. Let

$$
X \stackrel{u}{\rightarrow} Y \stackrel{v}{\rightarrow} Z \stackrel{w}{\rightarrow} S X
$$

be an AR-triangle. We write $(U, ?)$ for the cohomological functor $\operatorname{Hom}_{\mathcal{T}}(U, ?)$. Thus, we have a long exact sequence

$$
\left(U, S^{-1} Z\right) \stackrel{S^{-1} w_{*}}{\longrightarrow}(U, X) \stackrel{u_{*}}{\longrightarrow}(U, Y) \stackrel{v_{*}}{\longrightarrow}(U, Z) \stackrel{w_{*}}{\longrightarrow}(U, S X) .
$$

Let $S_{Z}(U)$ be the image of the map $w_{*}$. We have the exact sequence:

$$
0 \rightarrow S_{S^{-1} Z}(U) \rightarrow(U, X) \stackrel{u_{*}}{\longrightarrow}(U, Y) \stackrel{v_{*}}{\longrightarrow}(U, Z) \stackrel{w_{*}}{\longrightarrow} S_{Z}(U) \rightarrow 0 .
$$

Thus we have the equality

$$
\operatorname{dim}_{k} S_{Z}(U)+\operatorname{dim}_{k} S_{S^{-1} Z}(U)+\operatorname{dim}_{k}(U, Y)=\operatorname{dim}_{k}(U, X)+\operatorname{dim}_{k}(U, Z) .
$$

If $U$ is not isomorphic to $Z$, each map from $U$ to $Z$ is radical, thus $S_{Z}(U)$ is zero. If $U$ is isomorphic to $Z$, the map $w_{*}$ factors through the radical of $\operatorname{End}(Z)$, so $S_{Z}(Z)$ is isomorphic to $k$. Then summing the previous equality when $U$ runs over $\mathcal{S}$, we get

$$
\ell(X)+\ell(Z)=\ell(Y)+2 .
$$

Clearly $\ell$ is $\tau$-invariant, thus $\ell(Z)$ equals $\ell(X)$. If the decomposition of $Y$ is of the form $\bigoplus_{i=1}^{r} Y_{i}^{n_{i}}$, we get

$$
\ell(Y)=\sum_{i} n_{i} \ell\left(Y_{i}\right)=\sum_{i, X \rightarrow Y_{i} \in \tilde{\Gamma}} a_{X Y_{i}} \ell\left(Y_{i}\right)+a_{X X} \ell(X) .
$$

We deduce the formula

$$
2=\left(2-a_{X X}\right) \ell(X)-\sum_{i, X \rightarrow Y_{i} \in \tilde{\Gamma}} a_{X Y_{i}} \ell\left(Y_{i}\right) .
$$

Let $x$ be a vertex of the tree $\Delta$ and $\bar{x}$ its image in $\widetilde{\Gamma}$. Then an arrow $\bar{x} \rightarrow Y$ in $\widetilde{\Gamma}$ comes from an arrow $(x, 0) \rightarrow(y, 0)$ in $\mathbb{Z} \Delta$ or from an arrow $(x, 0) \rightarrow(y,-1)$ in $\mathbb{Z} \Delta$, i.e. from an arrow $(y, 0) \rightarrow(x, 0)$. Indeed the projection $\mathbb{Z} \Delta \rightarrow \mathbb{Z} \Delta / G$ is a covering. From this we deduce the equality

$$
2=\left(2-a_{\bar{x} \bar{x}}\right) d_{x}-\sum_{y, x \rightarrow y \in \Delta} a_{\bar{x} \bar{y}} d_{y}-\sum_{y, y \rightarrow x \in \Delta} a_{\bar{y} \bar{x}} d_{y}=\sum_{y \in \Delta_{0}} d_{y} C_{x y} .
$$


Now we can prove Theorem 4.1. The matrix $C$ is a 'generalized Cartan matrix'. The previous lemma gives us a subadditive function which is not additive. Thus by [23], the underlying graph of $C$ is of 'generalized Dynkin type'. As $C$ is symmetric, the graph is necessarily of type $\mathbb{A}, \mathbb{D}, \mathbb{E}$, or $\mathbb{L}$. But this graph is the graph $\Delta$ with the valuation $a$. We are done in the cases $\mathbb{A}, \mathbb{D}$, or $\mathbb{E}$.

The case $\mathbb{L}_{n}$ occurs when the AR-quiver contains at least one loop. We can see $\mathbb{L}_{n}$ as $\mathbb{A}_{n}$ with valuations on the vertices with a loop. Then, it is obvious that the automorphism groups of $\mathbb{Z} \mathbb{L}_{n}$ are generated by $\tau^{r}$ for an $r \geq 1$. But Proposition 4.2 tell us that a vertex $x$ with a loop satisfies $\tau x=x$. Thus $G$ is generated by $\tau$ and the AR-quiver has the following form:

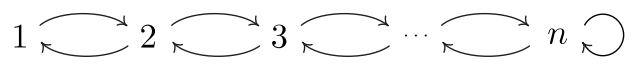

This quiver is isomorphic to the quiver $\mathbb{Z}_{\mathbb{A}_{2 n}} / G$ where $G$ is the group generated by the automorphism $\tau^{n} S=\rho$.

The suspension functor $S$ sends the indecomposables on indecomposables, thus it can be seen as an automorphism of the AR-quiver. It is exactly the automorphism $S$ defined in Section 2.2.

As shown in [49], it follows from the results of [30] that for each Dynkin tree $\Delta$ and for each weakly admissible group of automorphisms $G$ of $\mathbb{Z} \Delta$, there exists a locally finite triangulated category $\mathcal{T}$ such that $\Gamma_{\mathcal{T}} \simeq \mathbb{Z} \Delta / G$. This category is of the form $\mathcal{T}=\mathcal{D}^{b}(\bmod k \Delta) / \varphi$ where $\varphi$ is an auto-equivalence of $\mathcal{D}^{b}(\bmod k \Delta)$.

\section{Construction of a covering functor}

From now, we suppose that the AR-quiver $\Gamma$ of $\mathcal{T}$ is connected. We know its structure. It is natural to ask: Is the category $\mathcal{T}$ standard, i.e. equivalent as a $k$-linear category to the mesh category $k(\Gamma)$ ? First, in this part we construct a covering functor $F: k(\mathbb{Z} \Delta) \rightarrow \mathcal{T}$.

5.1. Construction. - We write $\pi: \mathbb{Z} \Delta \rightarrow \Gamma$ for the canonical projection. As $G$ is a weakly admissible group, this projection verifies the following property: if $x$ is a vertex of $\mathbb{Z} \Delta$, the number of arrows of $\mathbb{Z} \Delta$ with source $x$ is equal to the number of arrows of $\mathbb{Z} \Delta / G$ with source $\pi x$. Let $\mathcal{S}$ be a system of representatives of the isoclasses of indecomposables of $\mathcal{T}$. We write ind $\mathcal{T}$ for the full subcategory of $\mathcal{T}$ whose set of objects is $\mathcal{S}$. For a tree $\Delta$, we write $k(\mathbb{Z} \Delta)$ for the mesh category (see [39]). Using the same proof as Riedtmann [39], one shows the following theorem. 
TheOREM 5.1. - There exists a k-linear functor $F: k(\mathbb{Z} \Delta) \rightarrow$ ind $\mathcal{T}$ which is surjective and induces bijections:

$$
\bigoplus_{F z=F y} \operatorname{Hom}_{k(\mathbb{Z} \Delta)}(x, z) \longrightarrow \operatorname{Hom}_{\mathcal{T}}(F x, F y)
$$

for all vertices $x$ and $y$ of $\mathbb{Z} \Delta$.

5.2. Infinite case. - If the category $\mathcal{T}$ is locally finite not finite i.e. if there is infinitely many indecomposables, the constructed functor $F$ is immediately fully faithful. Thus we get the corollary.

COROLlary 5.2. - If ind $\mathcal{T}$ is not finite, then we have a $k$-linear equivalence between $\mathcal{T}$ and the mesh category $k(\mathbb{Z} \Delta)$.

5.3. Uniqueness criterion. - The covering functor $F$ can be seen as a $k$-linear functor from the derived category $\mathcal{D}^{b}(\bmod k \Delta)$ to the category $\mathcal{T}$. By construction, it satisfies the following property called the AR-property:

For each AR-triangle $X \stackrel{f}{\rightarrow} Y \stackrel{g}{\rightarrow} Z \stackrel{h}{\rightarrow} S X$ of $\mathcal{D}^{b}(\bmod k \Delta)$, there exists a triangle of $\mathcal{T}$ of the form $F X \stackrel{F f}{\longrightarrow} F Y \stackrel{F g}{\longrightarrow} F Z \stackrel{\epsilon}{\rightarrow} S F X$.

In fact, thanks to this property, $F$ is determined by its restriction to the subcategory $\operatorname{proj} k \Delta=k(\Delta)$, i.e. we have the following lemma.

Lemma 5.3. - Let $F$ and $G$ be $k$-linear functors from $\mathcal{D}^{b}(\bmod k \Delta)$ to $\mathcal{T}$. Suppose that $F$ and $G$ satisfy the AR-property and that the restrictions $F_{\mid k(\Delta)}$ and $G_{\mid k(\Delta)}$ are isomorphic. Then the functors $F$ and $G$ are isomorphic as $k$-linear functors.

Proof. - It is easy to construct this isomorphism by induction using the (TR3) axiom of the triangulated categories (see [36]).

\section{Particular cases of $k$-linear equivalence}

From now we suppose that the category $\mathcal{T}$ is finite, i.e. $\mathcal{T}$ has finitely many isoclasses of indecomposable objects.

TOME $135-2007-\mathrm{N}^{\mathrm{O}} 3$ 
6.1. Equivalence criterion. - Let $\Gamma$ be the AR-quiver of $\mathcal{T}$ and suppose that it is isomorphic to $\mathbb{Z} \Delta / G$. Let $\varphi$ be a generator of $G$. It induces an automorphism in the mesh category $k(\mathbb{Z} \Delta)$ that we still denote by $\varphi$. Then we have the following equivalence criterion.

Proposition 6.1. - The categories $k(\Gamma)$ and ind $\mathcal{T}$ are equivalent as $k$ categories if and only if there exists a covering functor $F: k(\mathbb{Z} \Delta) \rightarrow$ ind $\mathcal{T}$ and an isomorphism of functors $\Phi: F \circ \varphi \rightarrow F$.

The proof consists in constructing a $k$-linear equivalence between ind $\mathcal{T}$ and the orbit category $k(\mathbb{Z} \Delta) / \varphi^{\mathbb{Z}}$ using the universal property of the orbit category (see [30]), and then constructing an equivalence between $k(\mathbb{Z} \Delta) / \varphi^{\mathbb{Z}}$ and $k(\Gamma)$.

\subsection{Cylindric case for $\mathbb{A}_{n}$}

THEOREM 6.2. - If $\Delta=\mathbb{A}_{n}$ and $\varphi=\tau^{r}$ for some $r \geq 1$, then there exists a functor isomorphism $\Phi: F \circ \varphi \rightarrow F$, i.e. for each object $x$ of $k(\mathbb{Z} \Delta)$ there exists an automorphism $\Phi_{x}$ of $F x$ such that for each arrow $\alpha: x \rightarrow y$ of $\mathbb{Z} \Delta$, the following diagram commutes:

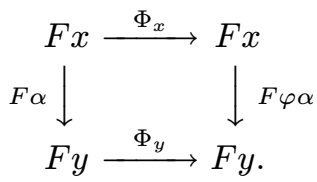

To prove this, we need the following lemma.

LemMA 6.3. - Let $\alpha: x \rightarrow y$ be an arrow of $\mathbb{Z} \mathbb{A}_{n}$ and let $c$ be a path from $x$ to $\tau^{r} y, r \in \mathbb{Z}$, which is not zero in the mesh category $k\left(\mathbb{Z} \mathbb{A}_{n}\right)$. Then $c$ can be written $c^{\prime} \alpha$ where $c^{\prime}$ is a path from $y$ to $\tau^{r} y$ (up to sign).

Proof of the lemma. - There is a path from $x$ to $\tau^{r} y$, thus, we have $\operatorname{Hom}_{k(\mathbb{Z} \Delta)}\left(x, \tau^{r} y\right) \simeq k$, and $x$ and $\tau^{r} y$ are opposite vertices of a 'rectangle' in $\mathbb{Z} \mathbb{A}_{n}$. This implies that there exists a path from $x$ to $\tau^{r} y$ beginning by $\alpha$.

Proof of Theorem 6.2. - Combining Proposition 6.1 and Lemma 5.3, we have just to construct an isomorphism between the restriction of $F$ and $F \circ \varphi$ to a subquiver $\mathbb{A}_{n}$.

Let us fix a full subquiver of $\mathbb{Z} \mathbb{A}_{n}$ of the following form

$$
x_{1} \stackrel{\alpha_{1}}{\longrightarrow} x_{2} \stackrel{\alpha_{2}}{\longrightarrow} \cdots \stackrel{\alpha_{n-1}}{\longrightarrow} x_{n}
$$

such that $x_{1}, \ldots, x_{n}$ are representatives of the $\tau$-orbits in $\mathbb{Z} \mathbb{A}_{n}$. We define the $\left(\Phi_{x_{i}}\right)_{i=1 \ldots n}$ by induction. We fix $\Phi_{x_{1}}=\operatorname{Id}_{F x_{1}}$. Now suppose we have 
constructed some automorphisms $\Phi_{x_{1}}, \ldots, \Phi_{x_{i}}$ such that for each $j \leq i$ the following diagram is commutative:

$$
\begin{gathered}
F x_{j-1} \stackrel{\Phi_{x_{j-1}}}{\longrightarrow} F x_{j-1} \\
F \alpha_{j-1} \downarrow \\
F x_{j} \stackrel{\Phi_{x_{j}}}{\longrightarrow} F x_{j} .
\end{gathered}
$$

The composition $\left(F \varphi \alpha_{i}\right) \circ \Phi_{x_{i}}$ is in the morphism space $\operatorname{Hom}_{\mathcal{T}}\left(F x_{i}, F x_{i+1}\right)$, which is isomorphic, by Theorem 5.1, to the space

$$
\bigoplus_{F z=F x_{i+1}} \operatorname{Hom}_{k(\mathbb{Z} \Delta)}\left(x_{i}, z\right) .
$$

Thus we can write

$$
\left(F \varphi \alpha_{i}\right) \Phi_{x_{i}}=\lambda F \alpha_{i}+\sum_{z \neq x_{i+1}} F \beta_{z}
$$

where $\beta_{z}$ belongs to $\operatorname{Hom}_{k(\mathbb{Z} \Delta)}\left(x_{i}, z\right)$ and $F z=F x_{i+1}$. But $F z$ is equal to $F x_{i+1}$ if and only if $z$ is of the form $\tau^{r \ell} x_{i+1}$ for an $\ell$ in $\mathbb{Z}$. By the lemma, we can write $\beta_{z}=\beta_{z}^{\prime} \alpha_{i}$. Thus we have the equality

$$
\left(F \varphi \alpha_{i}\right) \Phi_{x_{i}}=F\left(\lambda \operatorname{Id}_{x_{i+1}}+\sum_{z} \beta_{z}^{\prime}\right) F \alpha_{i}
$$

The scalar $\lambda$ is not zero. Indeed, $\Phi_{x_{i}}$ is an automorphism, thus the image of $\left(F \varphi \alpha_{i}\right) \Phi_{x_{i}}$ is not zero in the quotient

$$
\mathcal{R}\left(F x_{i}, F x_{i+1}\right) / \mathcal{R}^{2}\left(F x_{i}, F x_{i+1}\right) .
$$

Thus $\Phi_{x_{i+1}}=F\left(\lambda \operatorname{Id}_{x_{i+1}}+\sum_{z} \beta_{z}^{\prime}\right)$ is an automorphism of $F x_{i+1}$ which verifies the commutation relation

$$
\left(F \varphi \alpha_{i}\right) \circ \Phi_{x_{i}}=\Phi_{x_{i+1}} \circ F \alpha_{i} .
$$

6.3. Other standard cases. - In the mesh category $k(\mathbb{Z} \Delta)$, where $\Delta$ is a Dynkin tree, the length of the non zero paths is bounded. Thus there exist automorphisms $\varphi$ such that, for an arrow $\alpha: x \rightarrow y$ of $\Delta$, the paths from $x$ to $\varphi^{r} y$ vanish in the mesh category for all $r \neq 0$. In other words, for each arrow $\alpha: x \rightarrow y$ of $\mathbb{Z} \Delta$, we have

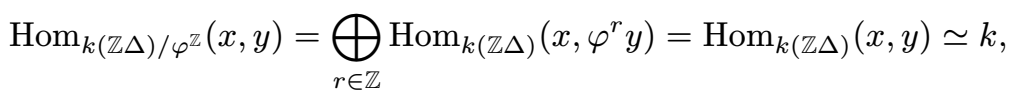

where $k(\mathbb{Z} \Delta) / \varphi^{\mathbb{Z}}$ is the orbit category (see Section 6.1).

TOME $135-2007-\mathrm{N}^{\mathrm{O}} 3$ 
Lemma 6.4. - Let $\mathcal{T}$ be a finite triangulated category with AR-quiver $\Gamma=$ $\mathbb{Z} \Delta / G$. Let $\varphi$ be a generator of $G$ and suppose that $\varphi$ verifies for each arrow $x \rightarrow y$ of $\mathbb{Z} \Delta$

$$
\bigoplus_{r \in \mathbb{Z}} \operatorname{Hom}_{k(\mathbb{Z} \Delta)}\left(x, \varphi^{r} y\right)=\operatorname{Hom}_{k(\mathbb{Z} \Delta)}(x, y) \simeq k .
$$

Let $F: k(\mathbb{Z} \Delta) \rightarrow \mathcal{T}$ and $G: k(\mathbb{Z} \Delta) \rightarrow \mathcal{T}$ be covering functors satisfying the $A R$-property. Suppose that $F$ and $G$ agree up to isomorphism on the objects of $k(\mathbb{Z} \Delta)$. Then $F$ and $G$ are isomorphic as $k$-linear functors.

Proof. - Using Lemma 5.3, we have just to construct an isomorphism between the functors restricted to $\Delta$. Let $\alpha: x \rightarrow y$ be an arrow of $\Delta$. Using Theorem 5.1 and the hypothesis, we have the isomorphisms

$$
\operatorname{Hom}_{\mathcal{T}}(F x, F y) \simeq \bigoplus_{F z=F y} \operatorname{Hom}_{k(\mathbb{Z} \Delta)}(x, z) \simeq \bigoplus_{r \in \mathbb{Z}} \operatorname{Hom}_{k(\mathbb{Z} \Delta)}\left(x, \varphi^{r} y\right) \simeq k
$$

and then

$$
\operatorname{Hom}_{\mathcal{T}}(G x, G y) \simeq \operatorname{Hom}_{\mathcal{T}}(F x, F y) \simeq k .
$$

Thus there exists a scalar $\lambda$ such that $G \alpha=\lambda F \alpha$. This scalar does not vanish since $F$ and $G$ are covering functors. As $\Delta$ is a tree, we can find some $\lambda_{x}$ for $x \in \Delta$ by induction such that

$$
G \alpha=\lambda_{x} \lambda_{y}^{-1} F \alpha .
$$

Now it is easy to check that $\Phi_{x}=\lambda_{x} \operatorname{Id}_{F x}$ is the functor isomorphism.

This lemma gives us an isomorphism between the functors $F$ and $F \circ \varphi$, Moreover, using the same argument, one can show that the covering functor $F$ is an $S$-functor and a $\tau$-functor.

For each Dynkin tree $\Delta$ we can determine the automorphisms $\varphi$ which satisfy this combinatorial property. Using the preceding lemma and the equivalence criterion we deduce the following theorem:

THEOREM 6.5. - Let $\mathcal{T}$ be a finite triangulated category with AR-quiver $\Gamma=$ $\mathbb{Z} \Delta / G$. Let $\varphi$ be a generator of $G$. If one of these cases holds,

- $\Delta=\mathbb{A}_{n}$ with $n$ odd and $G$ is generated by $\tau^{r}$ or $\varphi=\tau^{r} \phi$ with $r \geq \frac{1}{2}(n-1)$ and $\phi=\tau^{\frac{1}{2}(n+1)} S$;

- $\Delta=\mathbb{A}_{n}$ with $n$ even and $G$ is generated by $\rho^{r}$ with $r \geq n-1$ and $\rho=\tau^{\frac{1}{2} n} S$;

- $\Delta=\mathbb{D}_{n}$ with $n \geq 5$ and $G$ is generated by $\tau^{r}$ or $\tau^{r} \phi$ with $r \geq n-2$ and $\phi$ as in Theorem 2.1;

- $\Delta=\mathbb{D}_{4}$ and $G$ is generated by $\phi \tau^{r}$, where $r \geq 2$ and $\phi$ runs over $\sigma_{3}$; 
- $\Delta=\mathbb{E}_{6}$ and $G$ is generated by $\tau^{r}$ or $\tau^{r} \phi$ where $r \geq 5$ and $\phi$ is as in Theorem 2.1;

- $\Delta=\mathbb{E}_{7}$ and $G$ is generated by $\tau^{r}, r \geq 8$;

- $\Delta=\mathbb{E}_{8}$ and $G$ is generated by $\tau^{r}, r \geq 14$;

then $\mathcal{T}$ is standard, i.e. the categories $\mathcal{T}$ and $k(\Gamma)$ are equivalent as $k$-linear categories.

Corollary 6.6. - A finite maximal d-Calabi-Yau (see [30,8]) triangulated category $\mathcal{T}$, with $d \geq 2$, is standard, i.e. there exists a $k$-linear equivalence between $\mathcal{T}$ and the orbit category $\mathcal{D}^{b}(\bmod k \Delta) / \tau^{-1} S^{d-1}$ where $\Delta$ is Dynkin of type $\mathbb{A}, \mathbb{D}$ or $\mathbb{E}$

\section{Algebraic case}

For some automorphism groups $G$, we know the $k$-linear structure of $\mathcal{T}$. But what about the triangulated structure? We can only give an answer adding hypothesis on the triangulated structure. We distinguish two cases.

If $\mathcal{T}$ is locally finite, not finite, we have the following theorem which is proved in Section 7.2.

THEOREM 7.1. - Let $\mathcal{T}$ be a connected locally finite triangulated category with infinitely many indecomposables. If $\mathcal{T}$ is the base of a tower of triangulated categories [29], then $\mathcal{T}$ is triangle equivalent to $\mathcal{D}^{b}(\bmod k \Delta)$ for some Dynkin diagram $\Delta$.

Now if $\mathcal{T}$ is a finite standard category which is algebraic, i.e. $\mathcal{T}$ is triangle equivalent to $\underline{\mathcal{E}}$ for some $k$-linear Frobenius category $\mathcal{E}$ (see [31, 3.6]), then we have the following result which is proved in Section 7.3.

THEOREM 7.2. - Let $\mathcal{T}$ be a finite triangulated category, which is connected, algebraic and standard. Then, there exists a Dynkin diagram $\Delta$ of type $\mathbb{A}, \mathbb{D}$ or $\mathbb{E}$ and an auto-equivalence $\Phi$ of $\mathcal{D}^{b}(\bmod k \Delta)$ such that $\mathcal{T}$ is triangle equivalent to the orbit category $\mathcal{D}^{b}(\bmod k \Delta) / \Phi$.

This theorem combined with Corollary 6.6 yields the following result (compare to [30, Cor. 8.4]).

Corollary 7.3. - If $\mathcal{T}$ is a finite algebraic maximal d-Calabi-Yau category with $d \geq 2$, then $\mathcal{T}$ is triangle equivalent to the orbit category $\mathcal{D}^{b}(\bmod k \Delta) / S^{d} \nu^{-1}$ for some Dynkin diagram $\Delta$.

TOME $135-2007-\mathrm{N}^{\mathrm{O}} 3$ 
7.1. $\partial$-functor. - We recall the following definition from [29] and [46].

Definition 7.1.1. - Let $\mathcal{H}$ be an exact category and $\mathcal{T}$ a triangulated category. A $\partial$-functor $(I, \partial): \mathcal{H} \rightarrow \mathcal{T}$ is given by:

- an additive $k$-linear functor $I: \mathcal{H} \rightarrow \mathcal{T}$;

— for each conflation $\epsilon: X \stackrel{i}{\rightarrow} Y \stackrel{p}{\rightarrow} Z$ of $\mathcal{H}$, a morphism $\partial \epsilon: I Z \rightarrow S I X$ functorial in $\epsilon$ such that $I X \stackrel{I i}{\longrightarrow} I Y \stackrel{\ell I p}{\longrightarrow} I Z \stackrel{\partial \epsilon}{\longrightarrow} S I X$ is a triangle of $\mathcal{T}$.

For each exact category $\mathcal{H}$, the inclusion $I: \mathcal{H} \rightarrow \mathcal{D}^{b}(\mathcal{H})$ can be completed to a $\partial$-functor $(I, \partial)$ in a unique way. Let $\mathcal{T}$ and $\mathcal{T}^{\prime}$ be triangulated categories. If $(F, \varphi): \mathcal{T} \rightarrow \mathcal{T}^{\prime}$ is an $S$-functor and $(I, \partial): \mathcal{H} \rightarrow \mathcal{T}$ is a $\partial$-functor, we say that $F$ respects $\partial$ if $(F \circ I, \varphi(F \partial)): \mathcal{H} \rightarrow \mathcal{T}^{\prime}$ is a $\partial$-functor. Obviously each triangle functor respects $\partial$.

Proposition 7.4. - Let $\mathcal{H}$ be a k-linear hereditary abelian category and let $(I, \partial): \mathcal{H} \rightarrow \mathcal{T}$ be a $\partial$-functor. Then there exists a unique (up to isomorphism) $k$-linear $S$-functor $F: \mathcal{D}^{b}(\mathcal{H}) \rightarrow \mathcal{T}$ which respects $\partial$.

Proof. - On $\mathcal{H}$ (which can be seen as a full subcategory of $\mathcal{D}^{b}(\mathcal{H})$ ), the functor $F$ is uniquely determined. We want $F$ to be an $S$-functor, so $F$ is uniquely determined on $S^{n} \mathcal{H}$ for $n \in \mathbb{Z}$ too. Since $\mathcal{H}$ is hereditary, each object of $\mathcal{D}^{b}(\mathcal{H})$ is isomorphic to a direct sum of stalk complexes, i.e. complexes concentrated in a single degree. Thus, the functor $F$ is uniquely determined on the objects.

Now, let $X$ and $Y$ be stalk complexes of $\mathcal{D}^{b}(\mathcal{H})$ and $f: X \rightarrow Y$ a non-zero morphism. We can suppose that $X$ is in $\mathcal{H}$ and $Y$ is in $S^{n} \mathcal{H}$. If $n \neq 0,1$, $f$ is necessarily zero. If $n=0$, then $f$ is a morphism in $\mathcal{H}$ and $F f$ is uniquely determined. If $n=1, f$ is an element of $\operatorname{Ext}_{\mathcal{H}}^{1}\left(X, S^{-1} Y\right)$, so gives us a conflation $\epsilon: S^{-1} Y \stackrel{i}{\longmapsto} E \stackrel{p}{\rightarrow} X$ in $\mathcal{H}$. The functor $F$ respects $\partial$, thus $F f$ has to be equal to $\varphi \circ \partial \epsilon$ where $\varphi$ is the natural isomorphism between $S F S^{-1} Y$ and $F Y$. Since $\partial$ is functorial, $F$ is a functor. The result follows.

A priori this functor is not a triangle functor. We recall a theorem proved by B. Keller [29, Cor. 2.7].

TheOREM 7.5. - Let $\mathcal{H}$ be a $k$-linear exact category, and $\mathcal{T}$ be the base of a tower of triangulated categories [29]. Let $(I, \partial): \mathcal{H} \rightarrow \mathcal{T}$ be a $\partial$-functor such that for each $n<0$, and all objects $X$ and $Y$ of $\mathcal{H}$, the space $\operatorname{Hom}_{\mathcal{T}}\left(I X, S^{n} I Y\right)$ 
vanishes. Then there exists a triangle functor $F: \mathcal{D}^{b}(\mathcal{H}) \rightarrow \mathcal{T}$ such that the following diagram commutes up to isomorphism of $\partial$-functors:

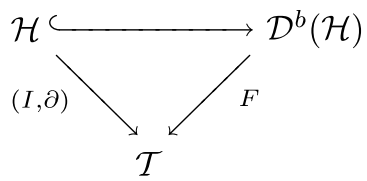

From Theorem 7.5, and the proposition above we deduce the following corollary.

Corollary 7.6 (compare [44]). - Let $\mathcal{T}, \mathcal{H}$ and $(I, \partial): \mathcal{H} \rightarrow \mathcal{T}$ be as in Theorem 7.5. If $\mathcal{H}$ is hereditary, then the unique functor $F: \mathcal{D}^{b}(\mathcal{H}) \rightarrow \mathcal{T}$ which respects $\partial$ is a triangle functor.

7.2. Proof of Theorem 7.1. - Let $F$ be the $k$-linear equivalence constructed in Theorem 5.1 between an algebraic triangulated category $\mathcal{T}$ and $\mathcal{D}^{b}(\mathcal{H})$ where $\mathcal{H}=\bmod k \Delta$ and $\Delta$ is a simply-laced Dynkin graph. As we saw in Section 6 , the covering functor is an $S$-functor.

The category $\mathcal{H}$ is the heart of the standard $t$-structure on $\mathcal{D}^{b}(\mathcal{H})$. The image of this $t$-structure through $F$ is a $t$-structure on $\mathcal{T}$. Indeed, $F$ is an $S$-equivalence, so the conditions (i) and (ii) from [7, Def. 1.3.1] hold obviously. And since $\mathcal{H}$ is hereditary, for an object $X$ of $\mathcal{D}^{b}(\mathcal{H})$, the morphism $\tau_{>0} X \rightarrow$ $S \tau_{\leq 0} X$ of the triangle

$$
\tau_{\leq 0} X \longrightarrow X \longrightarrow \tau_{>0} X \longrightarrow S \tau_{\leq 0} X
$$

vanishes. Thus the image of this triangle through $F$ is a triangle of $\mathcal{T}$ and condition (iii) of [7, Def. 1.3.1] holds. Then we get a $t$-structure on $\mathcal{T}$ whose heart is $\mathcal{H}$.

It results from [7, Prop. 1.2.4] that the inclusion of the heart of a $t$-structure can be uniquely completed to a $\partial$-functor. Thus we obtain a $\partial$-functor $\left(F_{0}, \partial\right)$ : $\mathcal{H} \rightarrow \mathcal{T}$ with $F_{0}=F_{\mid \mathcal{H}}$.

The functor $F$ is an $S$-equivalence. Thus for each $n<0$, and all objects $X$ and $Y$ of $\mathcal{H}$, the space $\operatorname{Hom}_{\mathcal{T}}\left(F X, S^{n} F Y\right)$ vanishes. Now we can apply Theorem 7.5 and we get the following commutative diagram

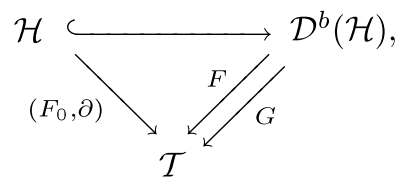

where $F$ is the $S$-equivalence and $G$ is a triangle functor. Note that a priori $F$ is an $S$-functor which does not respect $\partial$. The functors $F_{\mid \mathcal{H}}$ and $G_{\mid \mathcal{H}}$ are isomorphic. The functor $F$ is an $S$-functor thus we have an isomorphism 
$F_{\mid S^{n} \mathcal{H}} \simeq G_{\mid S^{n} \mathcal{H}}$ for each $n \in \mathbb{Z}$. Thus the functor $G$ is essentially surjective. Since $\mathcal{H}$ is the category $\bmod k \Delta$, to show that $G$ is fully faithful, we have just to show that for each $p \in \mathbb{Z}$, there is an isomorphism induced by $G$

$$
\operatorname{Hom}_{\mathcal{D}^{b}(\mathcal{H})}\left(A, S^{p} A\right) \stackrel{\sim}{\longrightarrow} \operatorname{Hom}_{\mathcal{T}}\left(G A, S^{p} G A\right)
$$

where $A$ is the free module $k \Delta$. For $p=0$, this is clear because $A$ is in $\mathcal{H}$. And for $p \neq 0$ both sides vanish.

Thus $G$ is a triangle equivalence between $\mathcal{D}^{b}(\mathcal{H})$ and $\mathcal{T}$.

7.3. Finite algebraic standard case. - For a small dg category $\mathcal{A}$, we denote by $\mathcal{C} \mathcal{A}$ the category of $\operatorname{dg} \mathcal{A}$-modules, by $\mathcal{D} \mathcal{A}$ the derived category of $\mathcal{A}$ and by per $\mathcal{A}$ the perfect derived category of $\mathcal{A}$, i.e. the smallest triangulated subcategory of $\mathcal{D} \mathcal{A}$ which is stable under passage to direct factors and contains the free $\mathcal{A}$-modules $\mathcal{A}($ ?,$A)$, where $A$ runs through the objects of $\mathcal{A}$. Recall that a small triangulated category is algebraic if it is triangle equivalent to per $\mathcal{A}$ for a $\operatorname{dg}$ category $\mathcal{A}$. For two small $\operatorname{dg}$ categories $\mathcal{A}$ and $\mathcal{B}$, a triangle functor per $\mathcal{A} \rightarrow$ per $\mathcal{B}$ is algebraic if it is isomorphic to the functor

$$
F_{X}=? \stackrel{L}{\otimes}_{\mathcal{A}} X
$$

associated with a dg bimodule $X$, i.e. an object of the derived category $\mathcal{D}\left(\mathcal{A}^{\mathrm{op}} \otimes \mathcal{B}\right)$.

Let $\Phi$ be an algebraic autoequivalence of $\mathcal{D}^{b}(\bmod k \Delta)$ such that the orbit category $\mathcal{D}^{b}(\bmod k \Delta) / \Phi$ is triangulated. Let $Y$ be a $\operatorname{dg} k \Delta-k \Delta$-bimodule such that $\Phi=F_{Y}$. In Section 9.3 of [30], it was shown that there is a canonical triangle equivalence between this orbit category and the perfect derived category of a certain small dg category. Thus, the orbit category is algebraic, and endowed with a canonical triangle equivalence to the perfect derived category of a small dg category. Moreover, by the construction in [loc. cit.], the projection functor

$$
\pi: \mathcal{D}^{b}(\bmod k \Delta) \longrightarrow \mathcal{D}^{b}(\bmod k \Delta) / \Phi
$$

is algebraic.

The proof of Theorem 7.0.5 is based on the following universal property of the triangulated orbit category $\mathcal{D}^{b}(\bmod k \Delta) / \Phi$. For the proof, we refer to Section 9.3 of [30].

Proposition 7.7. - Let $\mathcal{B}$ be a small dg category and

$$
F_{X}=? \stackrel{L}{\otimes_{k \Delta}} X: \mathcal{D}^{b}(\bmod k \Delta) \longrightarrow \operatorname{per} \mathcal{B}
$$

an algebraic triangle functor given by a $d g k \Delta$-A-bimodule $X$. Suppose that there is an isomorphism between $Y \otimes_{k \Delta}^{L} X$ and $X$ in the derived bimodule 
category $\mathcal{D}\left(k \Delta^{\mathrm{op}} \otimes \mathcal{B}\right)$. Then the functor $F_{X}$ factors, up to isomorphism of triangle functors, through the projection

$$
\pi: \mathcal{D}^{b}(\bmod k \Delta) \longrightarrow \mathcal{D}^{b}(\bmod k \Delta) / \Phi .
$$

Moreover, the induced triangle functor is algebraic.

Let us recall a lemma of Van den Bergh [33].

LEMmA 7.8. - Let $Q$ be a quiver without oriented cycles and $\mathcal{A}$ be a dg category. We denote by $k(Q)$ the category of paths of $Q$ and by $\mathrm{Can}: \mathcal{C} \mathcal{A} \rightarrow \mathcal{D} \mathcal{A}$ the canonical functor. Then we have the following properties:

a) Each functor $F: k(Q) \rightarrow \mathcal{D} \mathcal{A}$ lifts, up to isomorphism, to a functor $\widetilde{F}: k(Q) \rightarrow \mathcal{C A}$ which verifies the following property: For each vertex $j$ of $Q$, the induced morphism

$$
\bigoplus_{i} \widetilde{F} i \rightarrow \widetilde{F} j
$$

where $i$ runs through the immediate predecessors of $j$, is a monomorphism which splits as a morphism of graded $\mathcal{A}$-modules.

b) Let $F$ and $G$ be functors from $k(Q)$ to $\mathcal{C} \mathcal{A}$, and suppose that $F$ satisfies the property of a). Then any morphism of functors $\varphi:$ Can $\circ F \rightarrow$ Can $\circ G$ lifts to a morphism $\tilde{\varphi}: F \rightarrow G$.

Proof. - a) For each vertex $i$ of $Q$, the object $F i$ is isomorphic in $\mathcal{D} \mathcal{A}$ to its cofibrant resolution $X_{i}$. Thus for each arrow $\alpha: i \rightarrow j, F$ induces a morphism $f_{\alpha}: X_{i} \rightarrow X_{j}$ which can be lifted to $\mathcal{C} \mathcal{A}$ since the $X_{i}$ are cofibrant. Since $Q$ has no oriented cycle, it is easy to choose the $f_{\alpha}$ such that the property is satisfied.

b) For each vertex $i$ of $Q$, we may assume that $F i$ is cofibrant. Then we can lift $\varphi_{i}:$ Can $\circ F i \rightarrow$ Can $\circ G i$ to $\psi_{i}: F i \rightarrow G i$. For each arrow $\alpha$ of $Q$, the square

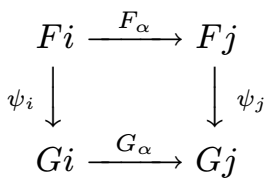

is commutative in $\mathcal{D} \mathcal{A}$. Thus the square

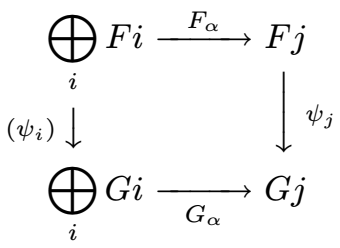

is commutative up to nullhomotopic morphism $h: \bigoplus_{i} F i \rightarrow G j$. Since the morphism $f: \bigoplus_{i} F i \rightarrow F j$ is split mono in the category of graded $\mathcal{A}$-modules, $h$ 
extends along $f$ and we can modify $\Psi_{j}$ so that the square becomes commutative in $\mathcal{C} \mathcal{A}$. The quiver $Q$ does not have oriented cycles, so we can construct $\widetilde{\varphi}$ by induction.

Proof of Theorem 7.2. - The category $\mathcal{T}$ is small and algebraic, thus we may assume that $\mathcal{T}=\operatorname{per} \mathcal{A}$ for some small $\operatorname{dg}$ category $\mathcal{A}$. Let $F: \mathcal{D}^{b}(\bmod k \Delta) \rightarrow$ $\mathcal{T}$ be the covering functor of Theorem 5.1. Let $\Phi$ be an auto-equivalence of $\mathcal{D}^{b}(\bmod k \Delta)$ such that the AR-quiver of the orbit category $\mathcal{D}^{b}(\bmod k \Delta) / \Phi$ is isomorphic (as translation quiver) to the AR-quiver of $\mathcal{T}$. We may assume that $\Phi=-\otimes_{k \Delta}^{L} Y$ for an object $Y$ of $\mathcal{D}\left(k \Delta^{\mathrm{op}} \otimes k \Delta\right)$. The orbit category $\mathcal{D}^{b}(\bmod k \Delta) / \Phi$ is algebraic, thus it is per $\mathcal{B}$ for some $\operatorname{dg}$ category $\mathcal{B}$.

The functor $F_{\mid k(\Delta)}$ lifts by Lemma 7.8 to a functor $\widetilde{F}$ from $k(\Delta)$ to $\mathcal{C} \mathcal{A}$. This means that the object $X=\widetilde{F}(k \Delta)$ has a structure of $\operatorname{dg} k \Delta^{\mathrm{op}} \otimes \mathcal{A}$-module. We denote by $X$ the image of this object in $\mathcal{D}\left(k \Delta^{\text {op }} \otimes \mathcal{A}\right)$.

The functors $F$ and $-\otimes_{k \Delta}^{L} X$ become isomorphic when restricted to $k(\Delta)$. Moreover $-\otimes_{k \Delta}^{L} X$ satisfies the AR-property since it is a triangulated functor. Thus by Lemma 5.3 , they are isomorphic as $k$-linear functors. So we have the following diagram:

$$
\begin{aligned}
& \mathcal{D}^{b}(\bmod k \Delta) \stackrel{-\stackrel{L}{\otimes}_{k \Delta} X}{\longrightarrow} \operatorname{per} \mathcal{A}=\mathcal{T} . \\
& \left(-\otimes_{k \Delta}^{L} Y\right.
\end{aligned}
$$

The category $\mathcal{T}$ is standard, thus there exists an isomorphism of $k$-linear functors

$$
c:-\stackrel{L}{\otimes}_{k \Delta} X \longrightarrow-\stackrel{L}{\otimes}_{k \Delta} Y \stackrel{L}{\otimes}_{k \Delta} X .
$$

The functor $-\otimes_{k \Delta}^{L} X$ restricted to the category $k(\Delta)$ satisfies the property of a) of Lemma 7.8. Thus we can apply b) and lift $c_{\mid k(\Delta)}$ to an isomorphism $\tilde{c}$ between $X$ and $Y \otimes_{k \Delta}^{L} X$ as dg- $k \Delta^{\text {op }} \otimes \mathcal{A}$-modules.

By the universal property of the orbit category, the bimodule $X$ endowed with the isomorphism $\tilde{c}$ yields a triangle functor from $\mathcal{D}^{b}(\bmod k \Delta) / \Phi$ to $\mathcal{T}$ which comes from a bimodule $Z$ in $\mathcal{D}\left(\mathcal{B}^{\text {op }} \otimes \mathcal{A}\right)$.

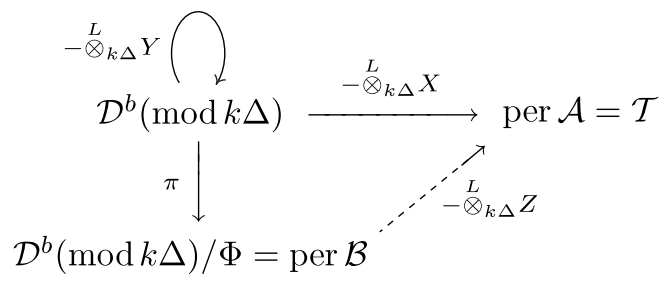


The functor $-\otimes_{k \Delta}^{L} Z$ is essentially surjective. Let us show that it is fully faithful. For $M$ and $N$ objects of $\mathcal{D}^{b}(\bmod k \Delta)$ we have the commutative diagram

$$
\operatorname{Hom}_{\mathcal{D} / \Phi}(\pi M, \pi N) \stackrel{\bigoplus_{n \in \mathbb{Z}} \operatorname{Hom}_{\mathcal{D}}\left(M, \Phi^{n} N\right)}{\stackrel{\stackrel{L}{\otimes}_{k \Delta} Z}{\longrightarrow}} \operatorname{Hom}_{\mathcal{T}}(F M, F N),
$$

where $\mathcal{D}$ means $\mathcal{D}^{b}(\bmod k \Delta)$. The two diagonal morphisms are isomorphisms, thus so is the horizontal morphism. This proves that $-\otimes_{k \Delta}^{L} Z$ is a triangle equivalence between the orbit category $\mathcal{D}^{b}(\bmod k \Delta) / \Phi$ and $\mathcal{T}$.

\section{Triangulated structure on the category of projectives}

Let $k$ be an algebraically closed field and $\mathcal{P}$ a $k$-linear category with split idempotents. The category $\bmod \mathcal{P}$ of contravariant finitely presented functors from $\mathcal{P}$ to $\bmod k$ is exact. As the idempotents split, the projectives of $\bmod \mathcal{P}$ coincide with the representables. Thus the Yoneda functor gives a natural equivalence between $\mathcal{P}$ and $\operatorname{proj} \mathcal{P}$. Assume besides that $\bmod \mathcal{P}$ has a structure of Frobenius category. The stable category $\underline{\bmod \mathcal{P}}$ is a triangulated category, we write $\Sigma$ for the suspension functor.

Let $S$ be an auto-equivalence of $\mathcal{P}$. It can be extended to an exact functor from $\bmod \mathcal{P}$ to $\bmod \mathcal{P}$ and thus to a triangle functor of $\bmod \mathcal{P}$. The aim of this part is to find a necessary condition on the functor $S$ such that the category $(\mathcal{P}, S)$ has a triangulated structure. Heller already showed [25, Thm. 16.4] that if there exists an isomorphism of triangle functors between $S$ and $\Sigma^{3}$, then $\mathcal{P}$ has a pretriangulated structure. But he did not succeed in proving the octahedral axiom. We are going to impose a stronger condition on the functor $S$ and prove the following theorem.

THEOREM 8.1. - Assume there exists an exact sequence of exact functors from $\bmod \mathcal{P}$ to $\bmod \mathcal{P}$ :

$$
0 \rightarrow \mathrm{Id} \longrightarrow X^{0} \longrightarrow X^{1} \longrightarrow X^{2} \longrightarrow S \rightarrow 0,
$$

where the $X^{i}, i=0,1,2$, take values in $\operatorname{proj} \mathcal{P}$. Then the category $\mathcal{P}$ has a structure of triangulated category with suspension functor $S$.

For an $M$ in $\bmod \mathcal{P}$, denote $T_{M}: X^{0} M \rightarrow X^{1} M \rightarrow X^{2} M \rightarrow S X^{0} M$ a standard triangle. A triangle of $\mathcal{P}$ will be a sequence $X: P \stackrel{u}{\rightarrow} Q \stackrel{v}{\rightarrow} R \stackrel{w}{\rightarrow} S P$ which is isomorphic to a standard triangle $T_{M}$ for an $M$ in $\bmod \mathcal{P}$.

TOME $135-2007-\mathrm{N}^{\mathrm{O}} 3$ 
8.1. S-complexes, $\Phi$-S-complexes and standard triangles. - Let $\mathcal{A} c p(\bmod \mathcal{P})$ be the category of acyclic complexes with projective components. It is a Frobenius category whose projective-injectives are the contractible complexes, i.e. the complexes homotopic to zero. The functor $Z^{0}: \mathcal{A c p}(\bmod \mathcal{P}) \rightarrow \bmod \mathcal{P}$ which sends a complex

$$
\cdots \longrightarrow X^{-1} \stackrel{x^{-1}}{\longrightarrow} X^{0} \stackrel{x^{0}}{\longrightarrow} X^{1} \stackrel{x^{1}}{\longrightarrow} \cdots
$$

to the kernel of $x^{0}$ is an exact functor. It sends the projective-injectives to projective-injectives and induces a triangle equivalence between $\mathcal{A} c p(\bmod \mathcal{P})$ and $\underline{\bmod } \mathcal{P}$.

Definition 8.1.1. - An object of $\mathcal{A} c p(\bmod \mathcal{P})$ is called an $S$-complex if it is $S$-periodic, i.e. if it has the following form:

$$
\cdots \longrightarrow P \stackrel{u}{\rightarrow} Q \stackrel{v}{\rightarrow} R \stackrel{w}{\rightarrow} S P \stackrel{S u}{\longrightarrow} S Q \longrightarrow \cdots .
$$

The category $S$-comp of $S$-complexes with $S$-periodic morphisms is a non full subcategory of $\mathcal{A} c p(\bmod \mathcal{P})$. It is a Frobenius category. The projectiveinjectives are the $S$-contractibles, i.e. the complexes homotopic to zero with an $S$-periodic homotopy. Using the functor $Z^{0}$, we get an exact functor from $S$-comp to $\bmod \mathcal{P}$ which induces a triangle functor

$$
\underline{Z^{0}}: \underline{S \text {-comp }} \longrightarrow \underline{\bmod } \mathcal{P} .
$$

Fix a sequence as in Theorem 8.1. Clearly, it induces for each object $M$ of $\underline{\bmod } \mathcal{P}$, a functorial isomorphism in $\underline{\bmod } \mathcal{P}, \Phi_{M}: \Sigma^{3} M \rightarrow S M$.

Let $Y$ be an $S$-complex:

$$
Y: \cdots \longrightarrow P \stackrel{u}{\rightarrow} Q \stackrel{v}{\rightarrow} R \stackrel{w}{\longrightarrow} S P \stackrel{S u}{\longrightarrow} S Q \longrightarrow \cdots
$$

Let $M$ be the kernel of $u$. Then $Y$ induces an isomorphism $\theta(\operatorname{in} \underline{\bmod } \mathcal{P})$ between $\Sigma^{3} M$ and $S M$. If $\theta$ is equal to $\Phi_{M}$, we will say that $X$ is a $\Phi$-S-complex.

Let $M$ be an object of $\bmod \mathcal{P}$. The standard triangle $T_{M}$ can be see as a $\Phi$-S-complex:

$$
\cdots \longrightarrow X^{0} M \longrightarrow X^{1} M \longrightarrow X^{2} M \longrightarrow S X^{0} M \longrightarrow S X^{1} M \longrightarrow \cdots
$$

The functor $T$ which sends an object $M$ of $\bmod \mathcal{P}$ to the $S$-complex $T_{M}$ is exact since the $X^{i}$ are exact. It satisfies the relation $Z^{0} \circ T \simeq \operatorname{Id}_{\bmod \mathcal{P}}$. Moreover, as it preserves the projective-injectives, it induces a triangle functor

$$
T: \underline{\bmod \mathcal{P}} \longrightarrow \underline{S \text {-comp }}
$$




\subsection{Properties of the functors $Z^{0}$ and $T$}

LEMMA 8.2. - An S-complex which is homotopy-equivalent to a $\Phi$-S-complex is a $\Phi$-S-complex.

Proof. - Let $X: P \stackrel{u}{\rightarrow} Q \stackrel{v}{\rightarrow} R \stackrel{w}{\rightarrow} S P$ be an $S$-complex homotopy-equivalent to the $\Phi$-S-complex $X^{\prime}: P^{\prime} \stackrel{u^{\prime}}{\longrightarrow} Q^{\prime} \stackrel{v^{\prime}}{\longrightarrow} R^{\prime} \stackrel{w^{\prime}}{\longrightarrow} S P^{\prime}$. Let $M$ be the kernel of $u$ and $M^{\prime}$ the kernel of $u^{\prime}$. By assumption, there exists a $S$-periodic homotopy equivalence $f$ from $X$ to $X^{\prime}$, which induces a morphism $g=Z^{0} f: M \rightarrow M^{\prime}$. Thus, we get the following commutative diagram:

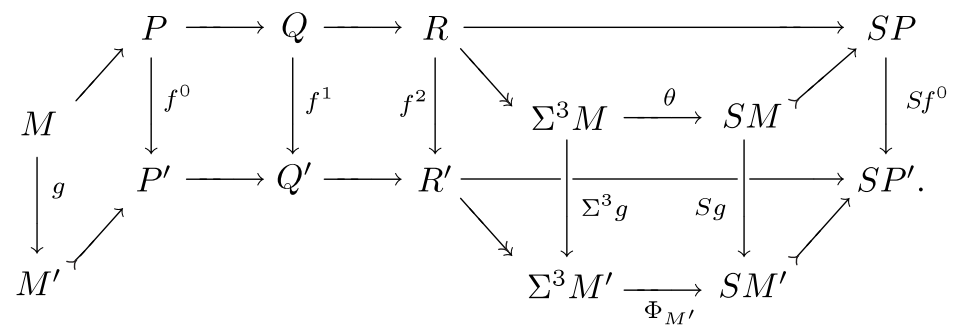

The morphism $g$ is an isomorphism of $\underline{\bmod \mathcal{P}}$ since $f$ is an isomorphism of $\underline{S \text {-comp. }}$. Thus the morphisms $\Sigma^{3} g$ and $S g$ are isomorphisms of $\underline{\bmod } \mathcal{P}$. The following equality in $\underline{\bmod } \mathcal{P}$

$$
\theta=(S g)^{-1} \Phi_{M^{\prime}} \Sigma^{3} g=\Phi_{M}
$$

shows that the complex $X$ is a $\Phi$-S-complex.

LEMMA 8.3. - Let

$$
\begin{gathered}
X: P \stackrel{u}{\rightarrow} Q \stackrel{v}{\rightarrow} R \stackrel{w}{\rightarrow} S P, \\
X^{\prime}: P^{\prime} \stackrel{u^{\prime}}{\longrightarrow} Q^{\prime} \stackrel{v^{\prime}}{\longrightarrow} R^{\prime} \stackrel{w^{\prime}}{\longrightarrow} S P^{\prime}
\end{gathered}
$$

be two $\Phi$-S-complexes. Suppose that we have a commutative square

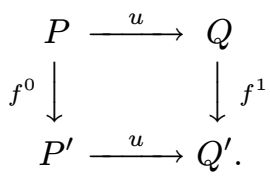

Then, there exists a morphism $f^{2}: R \rightarrow R^{\prime}$ such that $\left(f^{0}, f^{1}, f^{2}\right)$ extends to an $S$-periodic morphism from $X$ to $X^{\prime}$. 
Proof. - Let $M$ be the kernel of $u, M^{\prime}$ be the kernel of $u^{\prime}$ and $f: M \rightarrow M^{\prime}$ be the morphism induced by the commutative square. As $R$ and $R^{\prime}$ are projectiveinjective objects, we can find a morphism $g^{2}: R \rightarrow R^{\prime}$ such that the following square commutes:

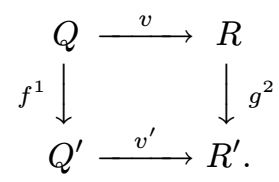

The morphism $g^{2}$ induces a morphism $g: S M \rightarrow S M^{\prime}$ such that the following square is commutative in $\underline{\bmod \mathcal{P}}$ :

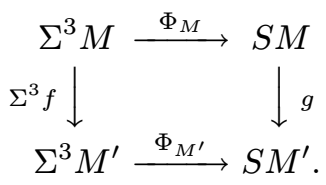

Thus the morphisms $S f$ and $g$ are equal in $\underline{\bmod } \mathcal{P}$, i.e. there exists a projectiveinjective $I$ of $\bmod \mathcal{P}$ and morphisms $\alpha: S M \rightarrow I$ and $\beta: I \rightarrow S M^{\prime}$ such that $g-S f=\beta \alpha$. Let $p$ (resp. $p^{\prime}$ ) be the epimorphism from $R$ onto $S M$ (resp. from $R^{\prime}$ onto $S M^{\prime}$ ). Then, as $I$ is projective, $\beta$ factors through $p^{\prime}$.

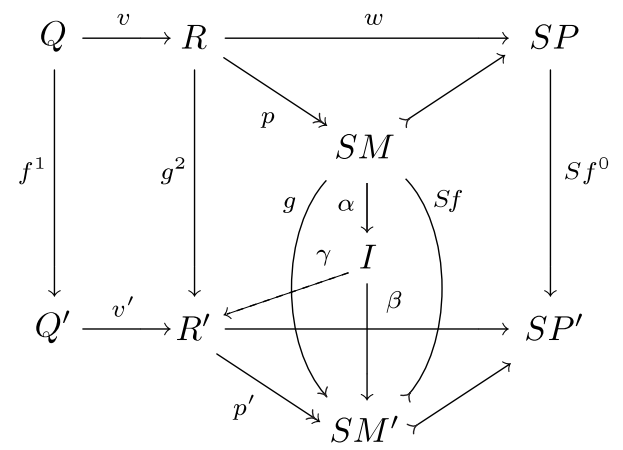

We put $f^{2}=g^{2}-\gamma \alpha p$. Then obviously, we have the equalities $f^{2} v=v^{\prime} f^{1}$ and $w^{\prime} f^{2}=S f^{0} w$. Thus the morphism $\left(f^{0}, f^{1}, f^{2}\right)$ extends to a morphism of $S$-comp.

Proposition 8.4. - The functor $\underline{Z^{0}}: \underline{\Phi-S-\operatorname{comp}} \rightarrow \underline{\bmod } \mathcal{P}$ is full and essentially surjective. Its kernel is an ideal whose square vanishes. 
Proof. - The functor $\underline{Z^{0}}$ is essentially surjective since we have the relation $\underline{Z^{0}} \circ \underline{T}=\operatorname{Id}_{\underline{\bmod } \mathcal{P}}$. Let us show that $\underline{Z^{0}}$ is full. Let

$$
\begin{aligned}
X: P \stackrel{u}{\rightarrow} Q \stackrel{v}{\rightarrow} R \stackrel{w}{\rightarrow} S P, \\
X^{\prime}: P^{\prime} \stackrel{u^{\prime}}{\longrightarrow} Q^{\prime} \stackrel{v^{\prime}}{\longrightarrow} R^{\prime} \stackrel{w^{\prime}}{\longrightarrow} S P^{\prime}
\end{aligned}
$$

be two $\Phi$ - $S$-complexes. Let $M$ (resp. $M^{\prime}$ ) be the kernel of $u\left(\right.$ resp. $u^{\prime}$ ). As $P$, $Q, P^{\prime}$ and $Q^{\prime}$ are projective-injective, there exist morphisms $f^{0}: P \rightarrow P^{\prime}$ and $f^{1}: Q \rightarrow Q^{\prime}$ such that the following diagram commutes:

$$
\begin{aligned}
& M \longmapsto P \stackrel{u}{\rightarrow} Q
\end{aligned}
$$

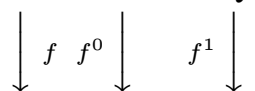

$$
\begin{aligned}
& M^{\prime} \longmapsto P^{\prime} \stackrel{u^{\prime}}{\longrightarrow} Q^{\prime} .
\end{aligned}
$$

Now the result follows from Lemma 8.3.

Now let $f: X \rightarrow X^{\prime}$ be a morphism in the kernel of $\underline{Z^{0}}$. Up to homotopy, we can suppose that $\underline{f}$ has the following form:

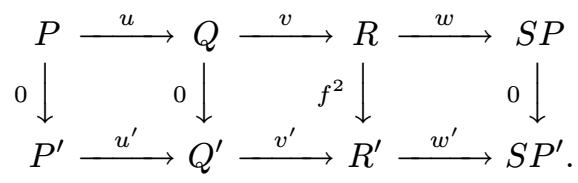

As the composition $w^{\prime} f^{2}$ vanishes and as $Q^{\prime}$ is projective-injective, $f^{2}$ factors through $v^{\prime}$. For the same argument, $f^{2}$ factors through $w$. If $f$ and $f^{\prime}$ are composable morphisms of the kernel of $\underline{Z^{0}}$, we get the following diagram:

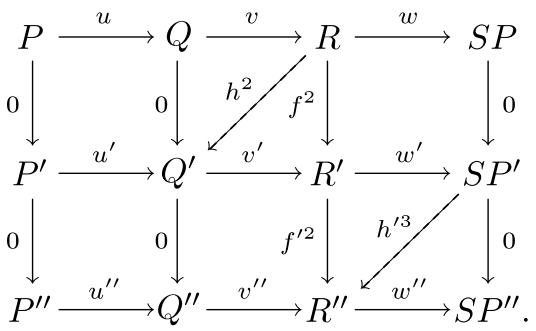

The composition $f^{\prime} \circ f$ vanishes obviously.

Corollary 8.5. - A $\Phi$-S-complex morphism $f$ which induces an isomorphism $\underline{Z^{0}}(f)$ in $\underline{\bmod } \mathcal{P}$ is an homotopy-equivalence.

This corollary comes from the previous theorem and from the following lemma.

TOME $135-2007-\mathrm{N}^{\circ} 3$ 
Lemma 8.6. - Let $F: \mathcal{C} \rightarrow \mathcal{C}^{\prime}$ be a full functor between two additive categories. If the kernel of $F$ is an ideal whose square vanishes, then $F$ detects isomorphisms.

Proof. - Let $u \in \operatorname{Hom}_{\mathcal{C}}(A, B)$ be a morphism in $\mathcal{C}$ such that $F u$ is an isomorphism. Since the functor $F$ is full, there exists $v$ in $\operatorname{Hom}_{\mathcal{C}}(B, A)$ such that $F v=(F u)^{-1}$. The morphism $w=u v-\operatorname{Id}_{B}$ is in the kernel of $F$, thus $w^{2}$ vanishes. Then the morphism $v\left(\operatorname{Id}_{B}-w\right)$ is a right inverse of $u$. In the same way we show that $u$ has a left inverse, so $u$ is an isomorphism.

Proposition 8.7. - The category of $\Phi$-S-complexes is equivalent to the category of $S$-complexes which are homotopy-equivalent to standard triangles.

Proof. - Since standard triangles are $\phi$-S-complexes, each $S$-complex that is homotopy equivalent to a standard triangle is a $\Phi$-S-complex (Lemma 8.2). Let $X: P \stackrel{u}{\rightarrow} Q \stackrel{v}{\rightarrow} R \stackrel{w}{\rightarrow} S P$ be a $\Phi$-S-complex. Let $M$ be the kernel of $u$. Then there exist morphisms $f^{1}: P \rightarrow X^{0} M$ and $f^{1}: Q \rightarrow X^{1} M$ such that the following diagram is commutative:

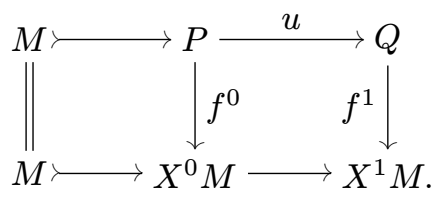

We can complete (Lemma 8.3) $f$ into an $S$-periodic morphism from $X$ in $T_{M}$. The morphism $f$ satisfies $Z^{0} f=\operatorname{Id}_{M}$, so $\underline{Z}^{0}\left(T_{M}\right)$ and $Z^{0}(X)$ are equal in $\bmod \mathcal{P}$. By the corollary, $T_{M}$ and $X$ are homotopy-equivalent. Thus the inclusion functor $T$ is essentially surjective.

These two diagrams summarize the results of this section:

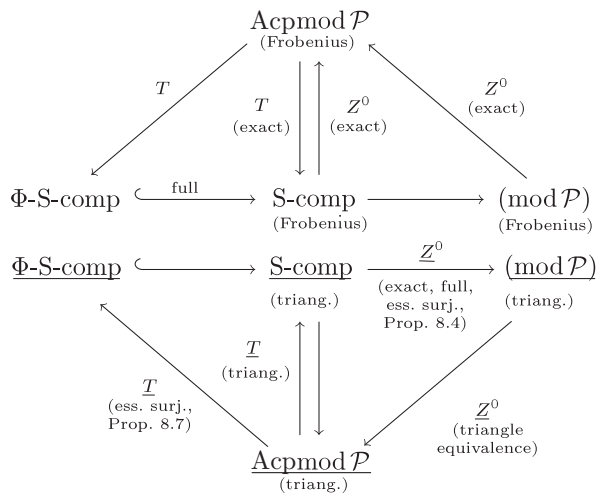


8.3. Proof of Theorem 8.1. - We are going to show that the $\Phi$ - $S$-complexes form a system of triangles of the category $\mathcal{P}$. We use triangle axioms as in [36].

TR0: For each object $M$ of $\mathcal{P}$, the $S$-complex $M=M \rightarrow 0 \rightarrow S M$ is homotopy-equivalent to the zero complex, so is a $\Phi$ - $S$-complex.

TR1: Let $u: P \rightarrow Q$ be a morphism of $\mathcal{P}$, and let $M$ be its kernel. We can find morphisms $f^{0}$ and $f^{1}$ so as to obtain a commutative square:

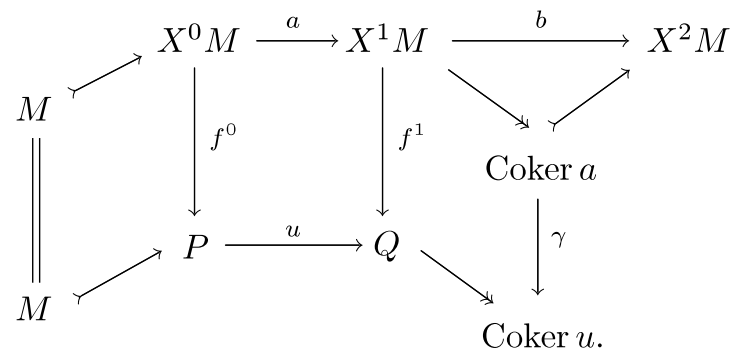

We form the push-out

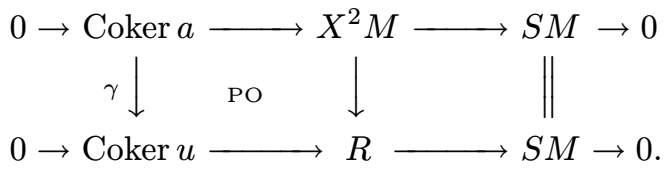

It induces a triangle morphism of the triangulated category $\underline{\bmod } \mathcal{P}$ :

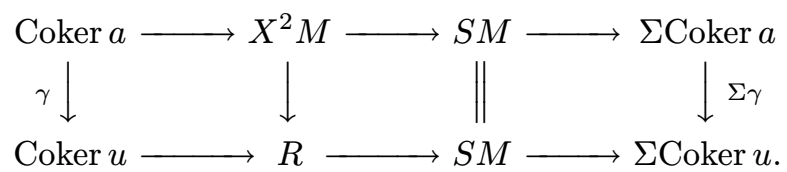

The morphism $\gamma$ is an isomorphism in $\bmod \mathcal{P}$ since Coker $a$ and Coker $u$ are canonically isomorphic to $\Sigma^{2} M$ in $\underline{\bmod } \mathcal{P}$. By the five lemma, $X^{2} M \rightarrow R$ is an isomorphism in $\underline{\bmod } \mathcal{P}$. Since $X^{2} M$ is projective-injective, so is $R$. Thus the complex $P \stackrel{u}{\rightarrow} Q \rightarrow R \rightarrow S P$ is an $S$-complex. Then we have to see that it is a $\Phi$-S-complex. Let $\theta$ be the isomorphism between $S M$ and $\Sigma^{3} M$ induced by this complex. We write $\alpha$ (resp. $\beta$ ) for the canonical isomorphism in $\underline{\bmod } \mathcal{P}$ between $\Sigma^{2} M$ and Coker $a$ (resp. Coker $u$ ). From the commutative diagram

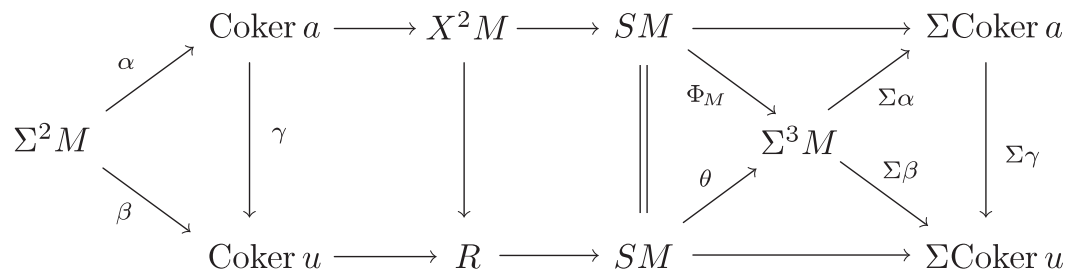

TOME $135-2007-\mathrm{N}^{\mathrm{O}} 3$ 
we deduce the equality $\theta=(\Sigma \beta)^{-1} \Sigma \gamma \Sigma \alpha \Phi_{M}=\Phi_{M}$ in $\underline{\bmod } \mathcal{P}$. The constructed $S$-complex is a $\Phi$-S-complex.

TR2: Let $X: P \stackrel{u}{\rightarrow} Q \stackrel{v}{\rightarrow} R \stackrel{w}{\rightarrow} S P$ be a $\Phi$-S-complex. It is homotopyequivalent to a standard triangle $T_{M}$. Thus the $S$-complex

$$
X^{\prime}: Q \stackrel{-v}{\longrightarrow} R \stackrel{-w}{\longrightarrow} S P \stackrel{-S u}{\longrightarrow} S Q
$$

is homotopy-equivalent to $T_{M}[1]$. Since $\underline{T}$ is a triangle functor, the objects $T_{\Sigma M}$ and $T_{M}[1]$ are isomorphic in the stable category $\underline{S-\text { comp }}$, i.e. they are homotopy-equivalent. Thus, by Lemma $8.2, T_{M}[1]$ is a $\Phi$-S-complex and then so is $X^{\prime}$.

TR3: This axiom is a direct consequence of Lemma 8.3.

TR4: Let $X$ and $X^{\prime}$ be two $\Phi$-S-complexes and suppose we have a commutative diagram:

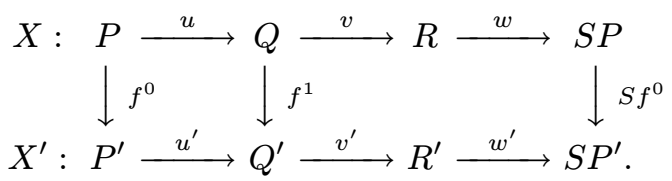

Let $M$ (resp. $M^{\prime}$ ) be the kernel of $u$ (resp. $u^{\prime}$ ), and $g: M \rightarrow M^{\prime}$ the induced morphism. The morphism $T g: T_{M} \rightarrow T_{M^{\prime}}$ induces a $S$-complex morphism $\widetilde{g}=\left(g^{0}, g^{1}, g^{2}\right)$ between $X$ and $X^{\prime}$.

We are going to show that we can find a morphism $f^{2}: R \rightarrow R^{\prime}$ such that $\left(f^{0}, f^{1}, f^{2}\right)$ can be extended in an $S$-complex morphism that is homotopic to $\widetilde{g}$. As $\left(g^{0}, g^{1}\right)$ and $\left(f^{0}, f^{1}\right)$ induce the same morphism $g$ in the kernels, we have some morphisms $h^{1}: Q \rightarrow P^{\prime}$ and $h^{2}: R \rightarrow Q^{\prime}$ such that $f^{0}-g^{0}=h^{1} u$ and $f^{1}-g^{1}=u^{\prime} h^{1}+h^{2} v$. We put $f^{2}=g^{2}+v^{\prime} h^{2}$. We have the equalities

$$
\begin{gathered}
f^{2} v=g^{2} v+v^{\prime} h^{2} v=v^{\prime}\left(g^{1}+h^{2} v\right)=v^{\prime}\left(f^{1}-u^{\prime} h^{1}\right)=v^{\prime} f^{1}, \\
w^{\prime} f^{2}=w^{\prime} g^{2}=\left(S g^{0}\right) w=\left(S f^{0}-S h^{1} S u\right) w=\left(S f^{0}\right) w .
\end{gathered}
$$

Thus $\left(f^{0}, f^{1}, f^{2}\right)$ can be extended to an $S$-periodic morphism $\tilde{f}$ which is $S$ homotopic to $\widetilde{g}$. Their respective cones $C(\widetilde{f})$ and $C(\widetilde{g})$ are isomorphic as $S$-complexes. Moreover, since $\widetilde{g}$ is a composition of $T g: T_{M} \rightarrow T_{M^{\prime}}$ with homotopy-equivalences, the cones $C(\widetilde{g})$ and $C(T g)$ are homotopy-equivalent.

In $\underline{\bmod } \mathcal{P}$, we have a triangle

$$
M \stackrel{g}{\rightarrow} M^{\prime} \longrightarrow C(g) \longrightarrow \Sigma M .
$$

Since $\underline{T}$ is a triangle functor, the sequence

$$
T_{M} \stackrel{T_{g}}{\longrightarrow} T_{M^{\prime}} \longrightarrow T_{C(g)} \longrightarrow T_{\Sigma M}
$$




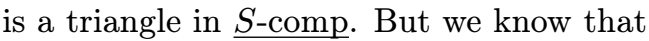

$$
T_{M} \stackrel{T g}{\longrightarrow} T_{M^{\prime}} \longrightarrow C(T g) \longrightarrow T_{M}[1]
$$

is a triangle in $S$-comp Thus the objects $C(T g)$ and $T_{C(g)}$ are isomorphic in $S$-comp i.e. homotopy-equivalent. Thus, the cone $C(\tilde{f})$ of $\tilde{f}$ is a $\Phi$-S-complex by Lemma 8.2.

\section{Application to the deformed preprojective algebras}

In this section, we apply Theorem 8.1 to show that the category of finite dimensional projective modules over a deformed preprojective algebra of generalized Dynkin type (see [8]) is triangulated. This will give us some examples of non standard triangulated categories with finitely many indecomposables.

9.1. Preprojective algebra of generalized Dynkin type. - Recall the notations of [8]. Let $\Delta$ be a generalized Dynkin graph of type $\mathbb{A}_{n}, \mathbb{D}_{n}(n \geq 4), \mathbb{E}_{n}(n=$ $6,7,8)$, or $\mathbb{L}_{n}$. Let $Q_{\Delta}$ be the following associated quiver:

$\Delta=\mathbb{A}_{n}(n \geq 1):$

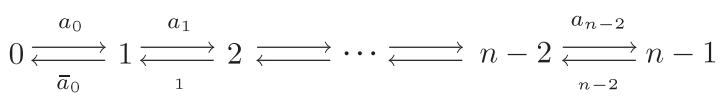

0

$\Delta=\mathbb{D}_{n}(n \geq 4):$

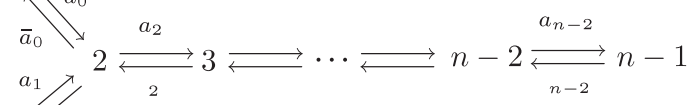

1

$\Delta=\mathbb{E}_{n}(n=6,7,8): \quad 1 \stackrel{a_{1}}{\rightleftarrows} 2 \underset{1}{\stackrel{a_{2}}{\rightleftarrows}} 3 \underset{3}{\rightleftarrows} 4 \rightleftarrows \cdots \underset{a_{3}}{\rightleftarrows} 4-2 \underset{n-2}{\rightleftarrows} n-1$

$\Delta=\mathbb{L}_{n}(n \geq 1): \quad \epsilon=\bar{\epsilon}\left(0 \underset{\bar{a}_{0}}{\stackrel{a_{0}}{\rightleftarrows}} \stackrel{a_{1}}{\rightleftarrows} 2 \rightleftarrows \cdots \rightleftarrows n-2 \underset{n-2}{\rightleftarrows} n-1\right.$.

The preprojective algebra $P(\Delta)$ associated to the graph $\Delta$ is the quotient of the path algebra $k Q_{\Delta}$ by the relations

$$
\sum_{s a=i} a \bar{a}, \quad \text { for each vertex } i \text { of } Q_{\Delta} .
$$

The following proposition is classical [8, Prop 2.1].

TOME $135-2007-\mathrm{N}^{\circ} 3$ 
Proposition 9.1. - The preprojective algebra $P(\Delta)$ is finite dimensional and selfinjective. Its Nakayama permutation $\nu$ is the identity for $\Delta=\mathbb{A}_{1}, \mathbb{D}_{2 n}$, $\mathbb{E}_{7}, \mathbb{E}_{8}$ and $\mathbb{L}_{n}$, and is of order 2 in all other cases.

9.2. Deformed preprojective algebras of generalized Dynkin type. - Let us recall the definition of deformed preprojective algebra introduced by [8]. Let $\Delta$ be a graph of generalized Dynkin type. We define an associated algebra $R(\Delta)$ as follows:

$$
\begin{gathered}
R\left(\mathbb{A}_{n}\right)=k, \quad R\left(\mathbb{D}_{n}\right)=k\langle x, y\rangle /\left(x^{2}, y^{2},(x+y)^{n-2}\right), \\
R\left(\mathbb{E}_{n}\right)=k\langle x, y\rangle /\left(x^{2}, y^{3},(x+y)^{n-3}\right), \quad R\left(\mathbb{L}_{n}\right)=k[x] /\left(x^{2 n}\right) .
\end{gathered}
$$

Further, we fix an exceptional vertex in each graph as follows (with the notations of the previous section):

$$
0 \text { for } \Delta=\mathbb{A}_{n} \text { or } \mathbb{L}_{n}, \quad 2 \text { for } \Delta=\mathbb{D}_{n}, \quad 3 \text { for } \Delta=\mathbb{E}_{n} .
$$

Let $f$ be an element of the square $\operatorname{rad}^{2} R(\Delta)$ of the radical of $R(\Delta)$. The deformed preprojective algebra $P^{f}(\Delta)$ is the quotient of the path algebra $k Q_{\Delta}$ by the relations

$$
\sum_{s a=i} a \bar{a}, \quad \text { for each non exceptional vertex } i \text { of } Q,
$$

and

$$
\begin{array}{ll}
a_{0} \bar{a}_{0} & \text { for } \Delta=\mathbb{A}_{n}, \\
\bar{a}_{0} a_{0}+\bar{a}_{1} a_{1}+a_{2} \bar{a}_{2}+f\left(\bar{a}_{0} a_{0}, \bar{a}_{1} a_{1}\right), \text { and }\left(\bar{a}_{0} a_{0}+\bar{a}_{1} a_{1}\right)^{n-2} & \text { for } \Delta=\mathbb{D}_{n}, \\
\bar{a}_{0} a_{0}+\bar{a}_{2} a_{2}+a_{3} \bar{a}_{3}+f\left(\bar{a}_{0} a_{0}, \bar{a}_{2} a_{2}\right), \text { and }\left(\bar{a}_{0} a_{0}+\bar{a}_{2} a_{2}\right)^{n-3} & \text { for } \Delta=\mathbb{E}_{n}, \\
\epsilon^{2}+a_{0} \bar{a}_{0}+\epsilon f(\epsilon), \text { and } \epsilon^{2 n} & \text { for } \Delta=\mathbb{L}_{n} .
\end{array}
$$

Note that if $f$ is zero, we get the preprojective algebra $P(\Delta)$.

9.3. Corollaries of [8]. - The following proposition [8, Prop. 3.4] shows that the category $\operatorname{proj} P^{f}(\Delta)$ of finite-dimensional projective modules over a deformed preprojective algebra satisfies the hypothesis of Theorem 8.1.

Proposition 9.2. - Let $A=P^{f}(\Delta)$ be a deformed preprojective algebra. Then there exists an exact sequence of $A$-A-bimodules

$$
0 \rightarrow{ }_{1} A_{\Phi^{-1}} \longrightarrow P_{2} \longrightarrow P_{1} \longrightarrow P_{0} \longrightarrow A \rightarrow 0,
$$

where $\Phi$ is an automorphism of $A$ and where the $P_{i}$ 's are projective as bimodules. Moreover, for each idempotent $e_{i}$ of $A$, we have $\Phi\left(e_{i}\right)=e_{\nu(i)}$.

So we can easily deduce the corollary: 
Corollary 9.3. - Let $P^{f}(\Delta)$ be a deformed preprojective algebra of generalized Dynkin type. Then the category proj $P^{f}(\Delta)$ of finite dimensional projective modules is triangulated. The suspension is the Nakayama functor.

Indeed, if $P_{i}=e_{i} A$ is a projective indecomposable, then $P_{i} \otimes_{A} A_{\Phi}$ is equal to $\Phi\left(e_{i}\right) A=e_{\nu(i)} A$ thus to $\nu\left(P_{i}\right)$.

Now we are able to answer to the question of the previous part and find a triangulated category with finitely many indecomposables which is not standard. The proof of the following theorem comes essentially from [8], Theorem 1.3.

THEOREM 9.4. - Let $k$ be an algebraically closed field of characteristic 2. Then there exist $k$-linear triangulated categories with finitely many indecomposables which are not standard.

Proof. - By [8, Thm. 1.3], we know that there exist basic deformed preprojective algebras of generalized Dynkin type $P^{f}(\Delta)$ which are not isomorphic to $P(\Delta)$. Thus the categories $\operatorname{proj} P^{f}(\Delta)$ and $\operatorname{proj} P(\Delta)$ can not be equivalent. But both are triangulated by Corollary 9.3 and have the same AR-quiver $\mathbb{Z} \Delta / \tau=Q_{\Delta}$.

Conversely, we have the following theorem:

Theorem 9.5. - Let $\mathcal{T}$ be a finite 1-Calabi-Yau triangulated category. Then $\mathcal{T}$ is equivalent to $\operatorname{proj} \Lambda$ as $k$-category, where $\Lambda$ is a deformed preprojective algebra of generalized Dynkin type.

Proof. - Let $M_{1}, \ldots, M_{n}$ be representatives of the isoclasses of indecomposable objects of $\mathcal{T}$. The $k$-algebra $\Lambda=\operatorname{End}\left(\bigoplus_{i=1}^{n} M_{i}\right)$ is basic, finite-dimensional and selfinjective since $\mathcal{T}$ has a Serre duality. It is easy to see that $\mathcal{T}$ and $\operatorname{proj} \Lambda$ are equivalent as $k$-categories.

Let $\bmod \Lambda$ be the category of finitely presented $\Lambda$-modules. It is a Frobenius category. Denote by $\Sigma$ the suspension functor of the triangulated category $\bmod \Lambda$. The category $\mathcal{T}$ is 1 -Calabi-Yau, that is to say that the suspension functor $S$ of the triangulated category $\mathcal{T}$ and the Serre functor $\nu$ are isomorphic. But in $\underline{\bmod } \Lambda$, the functors $S$ and $\Sigma^{3}$ are isomorphic. Thus, for each non projective simple $\Lambda$-module $M$ we have an isomorphism $\Sigma^{3} M \simeq \nu M$. We get immediately the result by $[8$, Thm. 1.2] .

TOME $135-2007-\mathrm{N}^{\mathrm{O}} 3$ 


\section{BIBLIOGRAPHY}

[1] H. AsASHIBA - "The derived equivalence classification of representationfinite selfinjective algebras", J. Algebra 214 (1999), p. 182-221.

[2] M. Auslander - "Functors and morphisms determined by objects", in Representation theory of algebras (Proc. Conf., Temple Univ., Philadelphia, Pa., 1976), Lecture Notes in Pure Appl. Math., vol. 37, Dekker, 1978, p. 1-244. Lecture Notes in Pure Appl. Math., Vol. 37.

[3] _ , "Isolated singularities and existence of almost split sequences", in Representation theory, II (Ottawa, Ont., 1984), Lecture Notes in Math., vol. 1178, Springer, 1986, p. 194-242.

[4] M. Auslander \& I. Reiten - "McKay quivers and extended Dynkin diagrams", Trans. Amer. Math. Soc. 293 (1986), p. 293-301.

[5] _ "Cohen-Macaulay and Gorenstein Artin algebras", in Representation theory of finite groups and finite-dimensional algebras (Bielefeld, 1991), Progr. Math., vol. 95, Birkhäuser, 1991, p. 221-245.

[6] R. Bautista, P. Gabriel, A. V. RoĬter \& L. Salmerón "Representation-finite algebras and multiplicative bases", Invent. Math. 81 (1985), p. 217-285.

[7] A. A. BeĬlinson, J. Bernstein \& P. Deligne - "Faisceaux pervers", in Analysis and topology on singular spaces, I (Luminy, 1981), Astérisque, vol. 100, Soc. Math. France, 1982, p. 5-171.

[8] J. BiaŁkowski, K. Erdmann \& A. Skowroński - "Deformed preprojective algebras of generalized Dynkin type", Trans. Amer. Math. Soc. 359 (2007), p. 2625-2650.

[9] J. BiaŁkowski \& A. Skowroński - "Calabi-Yau stable module categories of finite type", preprint http://www.mat.uni.torun.pl/ preprints/, 2006.

[10] _ "Nonstandard additively finite triangulated categories of CalabiYau dimension one in characteristic 3", to appear in Algebra and Discrete Mathematics.

[11] A. B. Buan, R. Marsh, M. Reineke, I. Reiten \& G. Todorov "Tilting theory and cluster combinatorics", Adv. Math. 204 (2006), p. 572618.

[12] P. Caldero, F. Chapoton \& R. Schiffler - "Quivers with relations arising from clusters $\left(A_{n}\right.$ case)", Trans. Amer. Math. Soc. 358 (2006), p. $1347-1364$.

[13] P. Caldero \& B. Keller - "From triangulated categories to cluster algebras", 2005. 
[14] E. Dieterich - "The Auslander-Reiten quiver of an isolated singularity", in Singularities, representation of algebras, and vector bundles (Lambrecht, 1985), Lecture Notes in Math., vol. 1273, Springer, 1987, p. 244-264.

[15] K. Erdmann \& N. Snashall - "On Hochschild cohomology of preprojective algebras. I, II", J. Algebra 205 (1998), p. 391-412, 413-434.

[16] _ "Preprojective algebras of Dynkin type, periodicity and the second Hochschild cohomology", in Algebras and modules, II (Geiranger, 1996), CMS Conf. Proc., vol. 24, Amer. Math. Soc., 1998, p. 183-193.

[17] P. GABRIEL \& A. V. RoĬTER - "Representations of finite-dimensional algebras", in Algebra, VIII, Encyclopaedia Math. Sci., vol. 73, Springer, 1992, With a chapter by B. Keller, p. 1-177.

[18] C. Geiss, B. Leclerc \& J. SchröER - "Auslander algebras and initial seeds for cluster algebras", preprint arXiv:math.RT/0506405, 2005.

[19] _ "Partial flag varieties and preprojective algebras", arXiv:math.RT/0609138, 2006.

[20] C. Geiss, B. LeClerC \& J. SCHRÖER - "Rigid modules over preprojective algebras", Invent. Math. 165 (2006), p. 589-632.

[21] D. HAPPEL - "On the derived category of a finite-dimensional algebra", Comment. Math. Helv. 62 (1987), p. 339-389.

[22] _ Triangulated categories in the representation theory of finitedimensional algebras, London Mathematical Society Lecture Note Series, vol. 119, Cambridge University Press, 1988.

[23] D. Happel, U. Preiser \& C. M. Ringel - "Binary polyhedral groups and Euclidean diagrams", Manuscripta Math. 31 (1980), p. 317-329.

[24] _ _ "Vinberg's characterization of Dynkin diagrams using subadditive functions with application to DTr-periodic modules", in Representation theory, II (Proc. Second Internat. Conf., Carleton Univ., Ottawa, Ont., 1979), Lecture Notes in Math., vol. 832, Springer, 1980, p. 280-294.

[25] A. Heller - "Stable homotopy categories", Bull. Amer. Math. Soc. 74 (1968), p. 28-63.

[26] T. Holm \& P. JøRGEnsen - "Cluster categories and selfinjective algebras: type A", preprint arXiv:math.RT/0610728, 2006.

[27] _ "Cluster categories and selfinjective algebras: type D", preprint arXiv:math.RT/0612451, 2006.

[28] O. IYAMA \& Y. Yoshino - "Mutations in triangulated categories and rigid Cohen-Macaulay modules", preprint arXiv:math.RT/0607736, 2006.

[29] B. KELlER - "Derived categories and universal problems", Comm. Algebra 19 (1991), p. 699-747.

[30] _ , "On triangulated orbit categories", Doc. Math. 10 (2005), p. 551581.

TOME $135-2007-\mathrm{N}^{\mathrm{O}} 3$ 
[31] _ "On differential graded categories", preprint arXiv:math.KT/0601185, 2006.

[32] B. Keller \& I. REITEN - "Cluster-tilted algebras are Gorenstein and stably Calabi-Yau", preprint arXiv:math.RT/0512471, 2005.

[33] _ "Acyclic Calabi-Yau categories", preprint arXiv:math.RT/0610594, 2006.

[34] B. Keller \& D. Vossieck - "Sous les catégories dérivées", C. R. Acad. Sci. Paris Sér. I Math. 305 (1987), p. 225-228.

[35] R. Marsh, M. Reineke \& A. Zelevinsky - "Generalized associahedra via quiver representations", Trans. Amer. Math. Soc. 355 (2003), p. 41714186.

[36] A. NeEman - Triangulated categories, Annals of Mathematics Studies, vol. 148, Princeton University Press, 2001.

[37] Y. PALU - "On algebraic Calabi-Yau categories", Ph.D. Thesis, in preparation.

[38] I. REITEN \& M. VAN DEN BERGH - "Noetherian hereditary abelian categories satisfying Serre duality", J. Amer. Math. Soc. 15 (2002), p. 295-366.

[39] C. Riedtmann - "Algebren, Darstellungsköcher, Überlagerungen und zurück", Comment. Math. Helv. 55 (1980), p. 199-224.

[40] _ _ "Representation-finite self-injective algebras of class $A_{n}$ ", in Representation theory, II (Proc. Second Internat. Conf., Carleton Univ., Ottawa, Ont., 1979), Lecture Notes in Math., vol. 832, Springer, 1980, p. 449-520.

[41] _ "Many algebras with the same Auslander-Reiten quiver", Bull. London Math. Soc. 15 (1983), p. 43-47.

[42] _ "Representation-finite self-injective algebras of class $D_{n}$ ", Compositio Math. 49 (1983), p. 231-282.

[43] C. M. RINGEL - Tame algebras and integral quadratic forms, Lecture Notes in Mathematics, vol. 1099, Springer, 1984.

[44] _ , "Hereditary triangulated categories", to appear in Comp. Math., 2006.

[45] G. TABUADA - "On the structure of Calabi-Yau categories with a cluster tilting subcategory", preprint arXiv:math.RT/0607394, 2006.

[46] J.-L. VERDIER - "Catégories dérivées. Quelques résultats", Lect. Notes Math. 569 (1977), p. 262-311.

[47] _ "Des catégories dérivées des catégories abéliennes", Astérisque (1996), p. 253 pp. (1997), With a preface by Luc Illusie, Edited and with a note by Georges Maltsiniotis.

[48] J. XiaO \& B. ZHU - "Relations for the Grothendieck groups of triangulated categories", J. Algebra 257 (2002), p. 37-50. 
[49] , "Locally finite triangulated categories", J. Algebra 290 (2005), p. $473-490$.

[50] Y. Yoshino - Cohen-Macaulay modules over Cohen-Macaulay rings, London Mathematical Society Lecture Note Series, vol. 146, Cambridge University Press, 1990. 